\title{
Trends in Mental Health Symptoms, Service Use, and Unmet Need for Services among US Adults through the First Nine Months of the COVID-19 Pandemic
}

Rebekah Coley ( $\square$ coleyre@bc.edu )

Boston College https://orcid.org/0000-0001-7172-061X

Christopher Baum

Boston College https://orcid.org/0000-0003-4766-3699

\section{Social Sciences - Article}

Keywords: COVID-19, mental health, anxiety, depression

Posted Date: January 19th, 2021

DOI: https://doi.org/10.21203/rs.3.rs-146306/v1

License: (c) (1) This work is licensed under a Creative Commons Attribution 4.0 International License. Read Full License

Version of Record: A version of this preprint was published at Translational Behavioral Medicine on October 18th, 2021. See the published version at https://doi.org/10.1093/tbm/ibab133. 


\section{Abstract}

The COVID-19 pandemic has led to rising morbidity, mortality, and social and economic disruption, stressors which have impaired mental health. 1 Evidence emerged of substantial increases in anxiety and depression in spring and summer, 2020.2-7 To understand the effects of the pandemic and inform public health initiatives, it is essential to track trends in mental health disorders, as well as use of and need for mental health services, and to identify demographic groups at highest risk. Analyzing cross-sectional samples of 1,483,378 US adults, we show that reports of anxiety and depression rose significantly from April to November, 2020 to rates six-times higher than early 2019 US norms. Use of prescription medication, counseling services, and unmet need for mental health services also rose significantly. Prevalence rates of mental health disorders were highest among young, less educated, single, female, Black, Hispanic, and other race/ethnicity adults, with age and education disparities growing over time. Hispanics, Blacks, other race/ethnicities, and less educated respondents also were significantly less likely to be receiving medication or counseling and reported higher unmet needs for services. Together, disparities in estimates of mental health disorders and mental health treatment indicate a striking disequilibrium between the potential need for and the use of mental health services during the COVID-19 pandemic. Rising mental health challenges are being born largely by young, less advantaged people of color and women, with the potential for expanded interruptions to optimal functioning and repercussions for social and economic recovery from COVID-19.

\section{Introduction}

Along with rising morbidity, mortality, and social and economic disruption, the COVID-19 pandemic also has brought a surge of mental health challenges. Stressors ranging from fear of illness, job and income loss, and social and educational disruptions have lead to rising stress and social isolation. ${ }^{1}$ Numerous single- or duo-wave surveys in spring and summer 2020 found increased levels of anxiety and depression in the US and other countries. ${ }^{2-7}$ One survey of US adults, for example, found rates of serious psychological distress of $14.2 \%$ in April and $13.0 \%$ in July, 2020, ${ }^{4}$ while a June, 2020 survey found prevalence rates of $24.3 \%$ for anxiety and $24.3 \%$ for depressive disorder, severely elevated compared to prior national norms. ${ }^{2}$

Due to demographic disparities in the physical, economic, and social impacts of COVID-19, it is likely that mental health disparities may emerge as well. Indeed, recent work found mental health challenges were heightened among younger adults, parents, Hispanic adults, and those of lower socioeconomic status early in the pandemic. ${ }^{1,2-3,7-8}$ Evidence to date has focused primarily on point-in-time or short-term shifts in mental health, presenting data from spring and summer 2020. It is essential to consider whether early increases in mental health symptomatology have remained stable, have grown, or have declined through the ongoing pandemic. Identifying the demographic groups at highest risk for increased mental health symptomatology is also critical.

In addition to tracking levels of psychological distress, to inform public health initiatives and direct scarce resources, we also consider changes in the use of and unmet need for mental health services. The COVID-19 pandemic led to guidelines to postpone non-essential medical services and screenings. ${ }^{9}$ Research is beginning to assess the growing gaps in mental health treatment in the US and internationally. ${ }^{10}$ An April, 
2020 survey of 880 US behavioral health organizations, for example, found that $93 \%$ had reduced services in response to COVID-19. ${ }^{11}$ An assessment of US electronic health records found mental and behavioral health visits declined in March, 2020, but dropped less than other types of medical visits, and rebounded more quickly to near pre-pandemic levels by April, $2020 .{ }^{12}$ It is essential to consider how changes in mental health care have evolved, and to evaluate the prevalence of unmet need for mental health services.

In the current research, we assess repeated cross-sectional surveys of nationally-representative samples of US residents from the US 2020 Household Pulse Survey conducted from April through November, 2020 to track trends in mental health disorder symptoms, mental health care, and unmet need for care across the entirety of the pandemic to date. These data provide a far more expansive view of changing mental health needs in the US population than prior short-term studies. The large and diverse sample also allows consideration of how trends vary across demographic strata.

\section{Results}

eTable 1 presents weighted sample descriptives. Table 2 shows odds ratios (OR), 95\% confidence intervals (Cl), and predicted prevalence rates (adjusted for cohort, state, and demographic characteristics) for anxiety and depression, with Figure 1 presenting prevalence rates across the sample cohorts. Table 3 and Figure 1 show these results for the prescription, counseling, and unmet need for counseling outcomes. Results for interaction models are presented in eTables 4-8. In describing the results in text, we focus on adjusted prevalence rates for ease of interpretability and practical significance.

\section{Anxiety}

Over the entire weighted sample, $44 \%$ of respondents met the criteria for anxiety disorder. Results from logistic regressions indicate significant growth in anxiety from early April through November (Table 2 and Figure 1), with prevalence rising from $38 \%$ for the first cohort (April 23-May 12, 2020) to $50 \%$ in the final cohort (November 11-23, 2020). Prevalence varied significantly by race/ethnicity, with anxiety higher among people identifying as other racial/ethnic groups (51\%), Hispanic (50\%), and Black (47\%) compared to Whites (41\%), who were higher than Asians (39\%). Interactions between race/ethnicity and wave were significant as a group (eTable 4), with gaps between Hispanics and Whites narrowing, and gaps between Asians and Whites growing across some cohorts.

Rates of anxiety were significantly higher among younger adults, with the prevalence among those age 18-29 (57\%) nearly double that of those aged 70 and above (30\%). Significant interaction results (eTable 5) found that age disparities grew over the cohorts, with anxiety prevalence rising most quickly for young adults. Females had significantly higher prevalence of anxiety than males ( $47 \%$ vs $40 \%$ ), and single adults with child(ren) $(51 \%)$ had higher prevalence than those in adult only households $(42 \%)$. Nonsignificant interactions showed stable sex and household structure differences over cohorts (eTables 6, 7). Anxiety prevalence declined significantly with greater education, ranging from $47 \%$ for those with a high school degree or less to $35 \%$ for respondents with a graduate degree. Significant interactions found that educational disparities grew in May and then stabilized (eTable 8). 


\section{Depression}

Over the entire analytic sample, $38 \%$ of respondents met the criteria for depressive disorder. Prevalence of depression grew significantly over the cohorts (Table 2 and Figure 1) from 32\% in late April/early May to $44 \%$ in mid November. Respondents identifying as other racial/ethnic backgrounds (45\%), Hispanics (44\%), and Blacks (43\%) reported higher prevalence of depression than Whites (35\%), with some narrowing of gaps between Hispanics and Whites across cohorts. Depression declined notably with age, with prevalence for 1829 year olds (52\%) nearly twice as high as those for $70+(27 \%)$. Age disparities grew significantly over time: a 20 point gap in the first cohort ( $42 \%$ vs $22 \%$ for $18-29$ vs $70+$ ) rose to a nearly 30 point gap by the final cohort (61\% to $32 \%)$. Females reported higher prevalence of depression than males (39\% vs $37 \%$ ), with nonsignificant shifts over cohorts. Prevalence of depression differed across all household structures, with respondents living with other adults reporting the lowest prevalence of depression, and single adults with child(ren) the highest prevalence at $45 \%$, differences which remained stable over cohorts. Depression declined with greater education, with prevalence dropping from $43 \%$ for those with a high school degree or less to $25 \%$ for respondents with a graduate degree. Education disparities grew over time, with the greatest growth in depression over cohorts among the least educated.

\section{Use of Prescription Medication}

Across the entire sample, $21 \%$ of respondents reported using prescription medication for emotional, behavioral, or mental health disorders in the prior month. Logistic regression models found that prevalence of medication use grew over time, particularly in late October and through November, ranging from $19 \%$ among the first cohort who reported on medication use in late August to $22 \%$ in mid November (Table 3 and Figure 1). Medication use varied by race/ethnicity, with prevalence among Whites $(23 \%)$ significantly higher than Hispanic (16\%), Black (15\%), and Asian (8\%) respondents. Prevalence of medication use varied significantly by age, ranging from a low of $17 \%$ among respondents aged 70 and above to a high of $22 \%$ among those in their 50 s. Females reported significantly higher medication use than males $(25 \%$ versus $15 \%)$. Adults living alone reported higher medication use (23\%) than those in multiple adult households $(20 \%)$, who in turn were higher than those in households with adults and child(ren) (19\%). In relation to education, respondents with some college reported significantly higher prevalence of medication use (22\%) than those with a high school degree or less (19\%). Sets of interactions were all nonsignificant, showing stable demographic differences across cohorts.

\section{Mental Health Counseling}

Over the whole sample, $9 \%$ of respondents reported accessing mental health counseling in the prior month. Logistic regression results (Table 3, Figure 1) found that prevalence increased over the cohorts, rising to $10 \%$ in mid October and November. Prevalence rates of mental health counseling were lower among Asian (6\%), Black (9\%), and Hispanic (9\%) respondents than their White (10\%) counterparts. Prevalence varied significantly by age as well, with $13 \%$ of $18-29$ year olds reporting counseling, more than three times higher than among respondents age 70 and above (4\%). Females were more likely than males to receive counseling (11\% versus $7 \%)$, as were those in single adult $(11 \%)$ or single adult with child(ren) $(13 \%)$ households compared to those living with other adults (9\%). Prevalence of mental health counseling was 
significantly lower among those with a high school degree or less (7\%) than among their peers with some college $(10 \%)$ or a bachelors or graduate degree (both $12 \%)$. Nonsignificant interactions indicated stable demographic differences across cohorts.

\section{Unmet Need for Mental Health Counseling}

Overall, $10 \%$ of the sample reported needing but not receiving mental health counseling services in the prior month. Logistic regression results (Table 3 and Figure 1) found increasing prevalence over cohorts, rising from $9 \%$ in late August to $11 \%$ starting in late October. Prevalence of unmet need for counseling were highest among those identifying as other race/ethnicity (16\%) and lowest among Asians (6\%), both significantly different than Whites $(10 \%)$. Unmet needs decreased significantly with age, with prevalence among young adults aged 18-29 (18\%) over five times higher than among the oldest respondents age 70 and above (3\%). Females were more likely than males to report unmet need for mental health counseling (13\% versus $8 \%)$. Significant but practically small differences emerged across household structure, while differences across educational strata were larger, with all respondents with higher education reporting greater prevalence of unmet need for mental health services than their peers with a high school degree or less (8\%), with the highest predicted rate $(13 \%)$ among those with some college. Like other measures of mental health services, nonsignificant interactions indicated stable demographic differences across cohorts.

\section{Intersections between Mental Health Disorders and Services}

A final set of descriptive analyses assessed intersections between mental health disorders and use of mental health services, using weighted chi square analyses. Of respondents meeting criteria for anxiety disorder, $35 \%$ reported use of prescription medication, $17 \%$ received counseling, and $24 \%$ reported unmet need for counseling versus $13 \%, 5 \%$, and $3 \%$ among those not meeting criteria (all $p<.001$ ). Of respondents meeting criteria for depressive disorder, $36 \%$ reported using medication, $17 \%$ received counseling, and $27 \%$ reported unmet need for counseling versus $14 \%, 6 \%$, and $4 \%$ among those not meeting criteria (all $p<.001$ ).

\section{Discussion}

The COVID-19 pandemic has disrupted nearly all aspects of life, leading to rising mortality rates, increasing economic inequities, and gross disturbances in people's daily lives and social interactions. Perhaps not surprisingly, these myriad stressors have led to rising rates of mental health disorder symptoms, a trend which was already apparent by April, 2020. ${ }^{2,7}$ Tracking trends in mental health disorder symptoms as well as use of mental health services over the course of the pandemic is essential to inform public health initiatives and identify subgroups of greatest concern.

Using nationally representative US Household Pulse survey data from nearly 1.5 million US residents between April and November, 2020, this study provides essential new insights into these questions. Results found significant increases in clinically-significant levels of anxiety through the first 9 months of the pandemic, with adjusted prevalence rising from $38 \%$ to $50 \%$ between April and November, 2020. By November, the prevalence of anxiety was over 6 times higher than national prevalence rates from JanuaryJune, 2019 (8\%) as reported in the NHIS survey. ${ }^{17}$ Rates of depression, which rose from $32 \%$ to $44 \%$ between 
April and November, 2020, similarly were more than 6 times higher than national norms (7\%) from early 2019. ${ }^{17}$ As such, these estimates present glaring evidence of growing mental health conditions and likely need for mental health services among US residents.

Use of mental health services, including prescription medication and counseling services, also showed growth (albeit at a less rapid rate) between April and November, 2020. Nationally, 22\% of US residents had taken medication and $10 \%$ had received counseling for mental health conditions in the prior month by November, 2020. In contrast to mental health disorders, these rates were less elevated in comparison to prior national norms (although different reporting periods prohibit exact comparisons). The 2019 NHIS surveys, for example, found $16 \%$ of adults had used prescription medication and $10 \%$ had received counseling for a mental health disorder in the prior year. ${ }^{18}$ Together, disparities in the estimates of mental health disorder and mental health treatment in 2020 indicate a striking disequilibrium between the potential need for and the use of mental health services. Indeed, by November, 2020, $11 \%$ of respondents reported needing but not receiving mental health services in the prior month, with prevalence of $24 \%$ and $27 \%$ for those meeting criteria for anxiety and depressive disorders, respectively, providing direct evidence of this disequilibrium.

Results from this study also extend evidence on notable disparities in mental health disorders and services across key demographic strata. Most notably, Hispanics, Blacks, and those identifying as other race/ethnicity, and those with a high school degree or less reported elevated prevalence of anxiety and depression in comparison to their White and more educated peers, but were significantly less likely to be receiving medication or counseling and reported higher unmet needs for mental health counseling. Females and single parents also reported heightened prevalence of anxiety and depression, as well as heightened prevalence of service receipt, although these groups still reported higher prevalence of unmet need for mental health counseling as well. Finally, young adults, those aged 18-29, reported rates of anxiety and depression at nearly twice the prevalence of older adults, and although the former were more likely to be receiving counseling, they also reported unmet need for such services at 6 times the prevalence of older adults. These patterns extend prior evidence of such disparities, 1,2-3,7-8 highlighting growth in age and educational disparities in mental health symptoms as the pandemic progressed.

Together, results provide the latest national evidence of striking increases in mental health challenges in the face of the COVID-19 pandemic, challenges born largely by young, less advantaged people of color and women. Disparities between prevalence rates of mental health disorders and receipt of services suggest that a substantial portion of US residents experiencing mental health symptoms are not receiving necessary services, with the potential for expanded interruptions to optimal functioning and related repercussions for social and economic recovery from the COVID-19 pandemic.

In interpreting these results, it is essential to acknowledge limitations, including cross-sectional data, brief (albeit validated) mental health screeners which do not specify clinical diagnoses, and online surveys which may miss key populations and lead to under-reporting of mental health symptoms. ${ }^{13}$ Beyond these key limitations, results suggest a growing mental health crisis driven by the COVID-19 pandemic. Assessing specific drivers of these increased mental health challenges derived from illness, financial, and social 
stressors will help to elucidate the potential for larger scale policy interventions, while evaluation of new modes of treatment, such as teletherapy, will help to direct future clinical service responses.

\section{References}

1. American Psychological Association. Stress in America 2020: a national mental health crisis. 2020;1-11. https://www.apa.org/news/press/releases/stress/2020/sia-mental-health-crisis.pdf. Accessed December 20, 2020

2. Czeisler ME Lane RI Petrosky E et al. Mental health, substance use, and suicidal ideation during the COVID-19 pandemic - United States, June 24-30, 2020. Centers for Disease Control and Prevention. MMWR. 2020;69(32):149-157.

3. Hyland P Shevlin M McBride $O$ et al. Anxiety and depression in the Republic of Ireland during the COVID19 pandemic. Acta Psych Scand. 2020;142(3):249-256. DOI: 10.1111/acps.13219.

4. McGinty EE Presskreischer R Anderson KE Han H Barry CL. Psychological distress and COVID-19-related stressors reported in a longitudinal cohort of 1337 US adults in April and July 2020. JAMA. 2020;324(24): 2555-2557. doi:10.1001/jama.2020.21231.

5. Petzold MB Bendau A Plag J. et al. Risk, resilience, psychological distress, and anxiety at the beginning of the COVID-19 pandemic in Germany. Brain Behav. 2020;10(9): e01745. doi: 10.1002/brb3.1745

6. Shi L Lu ZA Que JY et al. Prevalence of and risk factors associated with mental health symptoms among the general population in China during the Coronavirus disease 2019 pandemic. JAMA Netw Open. 2020 Jul 1;3(7):e2014053. doi:10.1001/jamanetworkopen.2020.14053

7. Twenge JM Joiner TE. Mental distress among U.S. adults during the COVID-19 pandemic. J Clin Psychol. 2020;76:2170-2182. DOI: 10.1002/jclp.23064.

8. American Psychological Association. Stress in America 2020: stress in the time of COVID-19. 2020;1:1-3. https://www.apa.org/news/press/releases/stress/2020/stress-in-america-covid.pdf. Accessed December 20, 2020.

9. Center for Medicare and Medicaid Services. Non-emergent, elective medical services, and treatment recommendations. 2020;1-2. https://www.cms.gov/files/document/cms-non-emergent-elective-medicalrecommendations.pdf. Accessed December 20, 2020.

10. World Health Organization. The impact of COVID-19 on mental, neurological and substance use services: results of a rapid assessment. 2020. https://www.who.int/publications/i/item/978924012455. Accessed December 20, 2020.

11. National Council for Behavioral Health. COVID-19 economic impact on behavioral health organizations. 2020. https://www.thenationalcouncil.org/wpcontent/uploads/2020/04/NCBH_COVID19_Survey_Findings_04152020.pdf?daf=375ateTbd56. Accessed December 20, 2020.

12. Ziedan E Simon KI Wing C. Effects of state COVID-19 closure policy on non-COVID-19 health care utilization. NBER Working Paper. 2020; 27621. Accessed January 1, 2021. http://www.nber.org/papers/w27621. 
13. Fields JF Hunter-Childs J Tersine A et al. Design and operation of the 2020 Household Pulse Survey, 2020. U.S. Census Bureau. Forthcoming. https://www2.census.gov/programs-surveys/demo/technicaldocumentation/hhp/2020_HPS_Background.pdf. Accessed November 1, 2020.

14. Kroenke K Spitzer RL Williams JB Löwe B. An ultra-brief screening scale for anxiety and depression: the PHQ-4. Psychos.2009:50:613-621. DOI: 1176/appi.psy.50.6.613

15. Kroenke K, Spitzer RL, Williams JBW, et al. Anxiety disorders in primary care: prevalence, impairment, comorbidity, and detection. Ann Intern Med. 2007;146:317-325. PMID: 17339617 DOI: 7326/0003-4819146-5-200703060-00004

16. Kroenke K, Spitzer RL, Williams JB: The Patient Health Questionnaire-2: validity of a two-item depression screener. MedCare. 2003;41:1284-1292. doi: 10.1097/01.MLR.0000093487.78664.3C

17. CDC, National Center for Health Statistics. Early release of selected mental health estimates based on data from the January-June 2019 National Health Interview Survey. 2020:1. Accessed December 20, 2020. https://www.cdc.gov/nchs/data/nhis/earlyrelease/ ERmentalhealth-508

18. Terlizzi EP Zablotsky B. Mental health treatment among adults: United States, 2019. NCHS Data Brief. 2020;380:1-8. Accessed January 2, 2021. https://www.cdc.gov/nchs/data/databriefs/db380-H.pdf

\section{Tables}


Table 2. Weighted logistic regression models predicting anxiety and depression.

\begin{tabular}{|c|c|c|c|c|}
\hline & Anxiety & & Depression & \\
\hline & OR $(95 \% \mathrm{Cl})$ & Pred. Rate & OR $(95 \% \mathrm{Cl})$ & Pred. Rate \\
\hline \multicolumn{5}{|l|}{ Race/Ethnicity } \\
\hline White, non Hispanic & Ref & 0.414 & Ref & 0.349 \\
\hline Black, non Hispanic & $1.093(1.061-1.126)$ & 0.470 & $1.229(1.192-1.267)$ & 0.430 \\
\hline Asian, non Hispanic & $0.835(0.799-0.872)$ & 0.388 & $1.020(0.975-1.067)$ & 0.354 \\
\hline Other, non Hispanic & $1.308(1.252-1.367)$ & 0.512 & $1.344(1.285-1.406)$ & 0.446 \\
\hline Hispanic & $1.103(1.070-1.137)$ & 0.496 & $1.155(1.120-1.191)$ & 0.442 \\
\hline \multicolumn{5}{|l|}{ Age } \\
\hline $18-29$ & Ref & 0.565 & Ref & 0.520 \\
\hline $30-39$ & $0.738(0.716-0.761)$ & 0.480 & $0.656(0.636-0.677)$ & 0.399 \\
\hline $40-49$ & $0.626(0.607-0.646)$ & 0.444 & $0.556(0.539-0.574)$ & 0.367 \\
\hline $50-59$ & $0.542(0.526-0.559)$ & 0.415 & $0.503(0.488-0.520)$ & 0.353 \\
\hline $60-69$ & $0.412(0.399-0.426)$ & 0.355 & $0.398(0.385-0.411)$ & 0.306 \\
\hline $70+$ & $0.316(0.304-0.328)$ & 0.296 & $0.339(0.325-0.352)$ & 0.271 \\
\hline \multicolumn{5}{|l|}{ Sex } \\
\hline Male & Ref & 0.402 & Ref & 0.365 \\
\hline Female & $1.384(1.360-1.408)$ & 0.471 & $1.177(1.156-1.199)$ & 0.393 \\
\hline \multicolumn{5}{|l|}{ Household structure } \\
\hline Adults only & Ref & 0.419 & Ref & 0.365 \\
\hline Single adult & $1.026(1.002-1.051)$ & 0.407 & $1.126(1.098-1.155)$ & 0.374 \\
\hline Single w/ child(ren) & $1.101(1.059-1.145)$ & 0.506 & $1.107(1.064-1.152)$ & 0.447 \\
\hline Adults w/ child(ren) & $0.978(0.956-0.999)$ & 0.465 & $0.940(0.919-0.962)$ & 0.395 \\
\hline \multicolumn{5}{|l|}{ Education } \\
\hline HS or below & Ref & 0.467 & Ref & 0.43 \\
\hline Some college & $0.898(0.879-0.918)$ & 0.460 & $0.832(0.814-0.850)$ & 0.403 \\
\hline Bachelors & $0.690(0.675-0.706)$ & 0.394 & $0.558(0.545-0.571)$ & 0.309 \\
\hline Grad degree & $0.631(0.616-0.646)$ & 0.347 & $0.470(0.458-0.482)$ & 0.252 \\
\hline \multicolumn{5}{|l|}{ Cohort } \\
\hline 23 Apr-12 May & Ref & 0.377 & Ref & 0.318 \\
\hline 14-26 May & $1.075(1.023-1.129)$ & 0.403 & $1.157(1.098-1.218)$ & 0.36 \\
\hline 28 May-9 Jun & $1.139(1.088-1.193)$ & 0.417 & $1.187(1.131-1.245)$ & 0.366 \\
\hline 11-23 Jun & $1.091(1.039-1.146)$ & 0.406 & $1.076(1.022-1.133)$ & 0.343 \\
\hline 25 Jun-7 Jul & $1.137(1.086-1.190)$ & 0.414 & $1.142(1.088-1.199)$ & 0.355 \\
\hline 9-21 Jul & $1.267(1.211-1.326)$ & 0.441 & $1.236(1.178-1.297)$ & 0.373 \\
\hline 19-31 Aug & $1.394(1.335-1.455)$ & 0.452 & $1.452(1.387-1.520)$ & 0.397 \\
\hline 2-14 Sep & $1.375(1.316-1.436)$ & 0.449 & $1.417(1.353-1.484)$ & 0.392 \\
\hline 16-28 Sep & $1.413(1.351-1.478)$ & 0.455 & $1.437(1.370-1.506)$ & 0.395 \\
\hline 30 Sep-12 Oct & $1.419(1.356-1.485)$ & 0.456 & $1.476(1.407-1.548)$ & 0.401 \\
\hline $14-26$ Oct & $1.471(1.404-1.541)$ & 0.464 & $1.541(1.468-1.618)$ & 0.410 \\
\hline 28 Oct-9 Nov & $1.733(1.645-1.826)$ & 0.502 & $1.731(1.639-1.827)$ & 0.435 \\
\hline $11-23 \mathrm{Nov}$ & $1.739(1.657-1.825)$ & 0.503 & $1.728(1.643-1.817)$ & 0.436 \\
\hline Constant & $0.944(0.879-1.013)$ & & $0.943(0.876-1.014)$ & \\
\hline Observations & 1,483,378 & & 1,483,378 & \\
\hline
\end{tabular}

Note. OR Odds Ratio. Cl Confidence Intervals. Models also adjust for state of residence. ORs and Cls in italics significant at $p<.01$. 
Table 3. Weighted logistic regression models predicting mental health services.

Prescription Medication Mental Health Counseling Unmet Need for Counseling

\begin{tabular}{ccccccr}
\hline & OR $(95 \% \mathrm{Cl})$ & Pred. Rate & OR $(95 \% \mathrm{Cl})$ & Pred. Rate & OR $(95 \% \mathrm{Cl})$ & Pred. Rate \\
\hline $\begin{array}{c}\text { Race/Ethnicity } \\
\text { White, non Hispanic }\end{array}$ & Ref & 0.228 & Ref & 0.097 & Ref & 0.099 \\
\hline Black, non Hispanic & $0.531(0.500-0.565)$ & 0.145 & $0.832(0.773-0.896)$ & 0.088 & $0.998(0.927-1.075)$ & 0.110 \\
\hline Asian, non Hispanic & $0.332(0.303-0.363)$ & 0.084 & $0.427(0.383-0.477)$ & 0.059 & $0.467(0.419-0.519)$ & 0.061 \\
\hline Other, non Hispanic & $0.959(0.888-1.035)$ & 0.225 & $1.081(0.977-1.196)$ & 0.117 & $1.388(1.275-1.511)$ & 0.156 \\
\hline Hispanic & $0.695(0.658-0.735)$ & 0.162 & $0.818(0.761-0.880)$ & 0.089 & $0.942(0.878-1.011)$ & 0.114 \\
\hline
\end{tabular}

Age

\begin{tabular}{ccccccc}
\hline $18-29$ & Ref & 0.191 & Ref & 0.134 & Ref & 0.178 \\
\hline $30-39$ & $1.127(1.065-1.193)$ & 0.203 & $0.950(0.892-1.013)$ & 0.131 & $0.829(0.780-0.882)$ & 0.146 \\
\hline $40-49$ & $1.167(1.104-1.233)$ & 0.208 & $0.774(0.726-0.826)$ & 0.106 & $0.607(0.569-0.647)$ & 0.110 \\
\hline $50-59$ & $1.174(1.111-1.239)$ & 0.219 & $0.584(0.546-0.625)$ & 0.086 & $0.441(0.412-0.472)$ & 0.088 \\
\hline $60-69$ & $1.003(0.949-1.060)$ & 0.207 & $0.366(0.340-0.394)$ & 0.059 & $0.253(0.236-0.273)$ & 0.055 \\
\hline $70+$ & $0.755(0.708-0.805)$ & 0.170 & $0.221(0.199-0.244)$ & 0.038 & $0.140(0.126-0.156)$ & 0.032 \\
\hline
\end{tabular}

Sex

\begin{tabular}{lcccccr} 
Male & Ref & 0.149 & Ref & 0.074 & Ref & 0.077 \\
\hline Female & $1.931(1.873-1.991)$ & 0.250 & $1.573(1.510-1.640)$ & 0.112 & $1.788(1.712-1.868)$ & 0.126
\end{tabular}

Household structure

\begin{tabular}{lcccccc} 
Adults only & Ref & 0.201 & Ref & 0.087 & Ref & 0.097 \\
\hline Single adult & $1.214(1.170-1.260)$ & 0.232 & $1.424(1.356-1.495)$ & 0.110 & $1.245(1.182-1.311)$ & 0.104 \\
\hline Single w/ child(ren) & $1.065(1.003-1.132)$ & 0.223 & $1.279(1.188-1.377)$ & 0.126 & $1.010(0.932-1.094)$ & 0.129 \\
\hline Adults w/ child(ren) & $0.907(0.874-0.941)$ & 0.192 & $0.884(0.841-0.928)$ & 0.097 & $0.830(0.790-0.873)$ & 0.109
\end{tabular}

Education

\begin{tabular}{lcccccr}
\hline HS or below & Ref & 0.186 & Ref & 0.066 & Ref & 0.083 \\
\hline Some college & $1.201(1.155-1.248)$ & 0.223 & $1.416(1.332-1.505)$ & 0.101 & $1.375(1.298-1.456)$ & 0.126 \\
\hline Bachelors & $1.029(0.990-1.071)$ & 0.199 & $1.579(1.486-1.678)$ & 0.116 & $1.160(1.094-1.229)$ & 0.110 \\
\hline Grad degree & $1.049(1.008-1.092)$ & 0.198 & $1.849(1.740-1.965)$ & 0.120 & $1.177(1.108-1.249)$ & 0.095
\end{tabular}

Cohort

\begin{tabular}{lcccccc} 
19-31 Aug & Ref & 0.194 & Ref & 0.087 & Ref & 0.092 \\
\hline 2-14 Sep & $1.018(0.973-1.066)$ & 0.196 & $1.042(0.978-1.110)$ & 0.091 & $1.062(0.997-1.132)$ & 0.097 \\
\hline 16-28 Sep & $1.032(0.986-1.081)$ & 0.198 & $1.039(0.975-1.107)$ & 0.090 & $1.160(1.085-1.240)$ & 0.104 \\
\hline
\end{tabular}




\begin{tabular}{ccccccc}
\hline 30 Sep-12 Oct & $1.008(0.962-1.057)$ & 0.195 & $1.038(0.975-1.106)$ & 0.090 & $1.071(1.004-1.141)$ & 0.097 \\
\hline $14-26$ Oct & $1.057(1.006-1.111)$ & 0.202 & $1.162(1.083-1.246)$ & 0.100 & $1.176(1.095-1.263)$ & 0.106 \\
\hline 28 Oct-9 Nov & $1.089(1.029-1.152)$ & 0.208 & $1.138(1.057-1.226)$ & 0.098 & $1.275(1.182-1.376)$ & 0.112 \\
\hline $11-23$ Nov & $1.147(1.089-1.209)$ & 0.216 & $1.152(1.072-1.236)$ & 0.099 & $1.259(1.173-1.351)$ & 0.111 \\
\hline Constant & $0.219(0.196-0.244)$ & & $0.073(0.062-0.088)$ & & $0.124(0.107-0.143)$ & 0.092 \\
\hline
\end{tabular}

Observations

528,615

528,586

528,925

Note. OR Odds Ratio. Cl Confidence Intervals. Models also adjust for state of residence. ORs and Cls in italics significant at $p<.01$.

\section{Methods}

\section{Survey Data}

We drew data from all available cohorts of the 2020 Household Pulse Survey, conducted by the U.S. Census Bureau and other government agencies to track effects of COVID-19 on US residents. ${ }^{13}$ These data are publicly available through the US Census bureau [https://www.census.gov/programs-surveys/householdpulse-survey/datasets.html]. Pulse conducted on-line surveys with cross-sections of adults in American households across all 50 states and Washington, DC in weekly or biweekly samples drawn from April 23 through July 21 (Phase 1), August 19 through October 26 (Phase 2), and October 28 through December 21, 2020 (Phase 3; data through November 23 were available for analysis). A small proportion of respondents repeated surveys for one or two additional weeks; we included each respondent's first survey data only. Data were clustered into approximately 2 -week cohorts, with a total sample of 1,483,378 unique respondents. Sample weights adjust for nonresponse and sampling stratification to produce estimates representative of the US adult population. ${ }^{13}$ Our university Institutional Review Board reviewed this study and considered it exempt.

\section{Mental Health Reports}

Respondents completed the widely-used and validated Patient Health Questionnaire for Depression and Anxiety, ${ }^{14}$ encompassing the 2 -item Generalized Anxiety Disorder Screener (GAD-2) ${ }^{15}$ to report symptoms of anxiety ("feeling nervous, anxious, or on edge"; "not being able to stop or control worrying", summed) and the 2-item Patient Health Questionnaire (PHQ-2) ${ }^{16}$ to report symptoms of depression ("feeling down, depressed, or hopeless"; "having little interest or pleasure in doing things", summed) in the past week ( 0 "not at all" to 3 "nearly every day"). Based on validation studies, ${ }^{14-16}$ responses $\geq 3$ were delineated as clinically significant indicators of likely anxiety disorder and depressive disorder, respectively. Starting in Phase 2, respondents also reported whether (yes/no) in the past 4 weeks they took prescription medication for mental health needs; received counseling or therapy from a mental health professional; and needed but did not receive counseling or therapy from a mental health professional, questions drawn from the National Health Interview Survey (NHIS).

\section{Demographics}


Respondents reported on their birth year (coded into age cohorts of 18-29; 30-39; 40-49; 50-59; 60-69; 70+), sex (male, female), race and ethnicity (coded non-Hispanic White; non-Hispanic Black; non-Hispanic Asian; non-Hispanic other; or Hispanic of any race), family structure (coded single adult; multiple adults; single adult with child(ren); multiple adults with child(ren)), and educational attainment (coded high school degree or less; some college; bachelors degree; graduate degree). Missing demographic data were imputed using hot deck imputation by the U.S. Census Bureau. ${ }^{13}$ State of residence was also recorded.

\section{Statistical analyses}

To assess trends over time in mental health symptoms and treatment, we conducted logistic regression models on each of the five mental health measures, using Stata version 16.1. All models included age, sex, race/ethnicity, family structure, and education; cohort indicators to assess shifts over time; state indicators to adjust for geographic differences; and clustered standard errors to properly estimate variances. We then interacted each set of demographic variables with the cohort indicators to assess whether time trends varied across subgroups of the US population, assessing the omnibus significance of each set of interactions using Wald tests. Two-tailed significance tests were employed, adjusted for multiple comparisons with Bonferroni corrections (thus considering $\mathrm{p}<.01$ as statistically significant). All analyses incorporated population weights to make the samples nationally representative. ${ }^{13}$

\section{Declarations}

Acknowledgements

Author Contributions:

Coley: concept and design; interpretation of data; drafting of manuscript; revision and approval of manuscript

Baum: concept and design; acquisition and analysis of data; interpretation of data; revision and approval of manuscript.

Conflict of Interest Disclosures: Coley and Baum have no conflicts to disclose.

Funding/Support: This work was completed using publicly available data from the US Household Pulse Survey, conducted by the US Census Bureau and other federal agencies, and was supported through a grant from Boston College (Abuelezam, Baum, Coley, Fusaro, Gates, Hawkins, Olivetti, Yadama co-PIs) entitled Data Analytics for Social Impact.

Role of the Funder: The funder had no role in the design, conduct, or completion of the study.

Disclaimer: The findings and conclusions in this study are those of the authors and do not necessarily represent the official position of the US Census Bureau or other federal agencies.

Additional Contributions: We thank Summer Sherburne Hawkins, PhD MS, Boston College for feedback on the manuscript and Naoka E. Carey, JD, Boston College for support in reviewing the literature. 
Etables

Page $13 / 28$ 
eTable 1. Weighted sample descriptives from the US Household Pulse Survey, N=1,483,378. ${ }^{a}$

\begin{tabular}{|c|c|c|c|}
\hline & Weight & & Weighted $\%$ \\
\hline Mental Health Outcomes & & State & \\
\hline Anxiety & $43.8 \%$ & Alabama & $1.5 \%$ \\
\hline Depression & $37.9 \%$ & Alaska & $0.2 \%$ \\
\hline Prescription medication & $20.1 \%$ & Arizona & $2.2 \%$ \\
\hline Counseling services & $9.4 \%$ & Arkansas & $0.9 \%$ \\
\hline Unmet need for services & $10.3 \%$ & California & $12.2 \%$ \\
\hline Race/Ethnicity & & Colorado & $1.8 \%$ \\
\hline White, non Hispanic & $61.2 \%$ & Connecticut & $1.1 \%$ \\
\hline Black, non Hispanic & $12.1 \%$ & Delaware & $0.3 \%$ \\
\hline Asian, non Hispanic & $5.2 \%$ & District of Columbia & $0.2 \%$ \\
\hline Other, non Hispanic & $3.8 \%$ & Florida & $6.9 \%$ \\
\hline Hispanic & $17.7 \%$ & Georgia & $3.2 \%$ \\
\hline Age & & Hawaii & $0.4 \%$ \\
\hline $18-29$ & $19.4 \%$ & Idaho & $0.5 \%$ \\
\hline $30-39$ & $19.1 \%$ & Illinois & $3.8 \%$ \\
\hline $40-49$ & $16.6 \%$ & Indiana & $2.0 \%$ \\
\hline $50-59$ & $16.7 \%$ & lowa & $0.9 \%$ \\
\hline $60-69$ & $16.6 \%$ & Kansas & $0.8 \%$ \\
\hline $70+$ & $11.6 \%$ & Kentucky & $1.3 \%$ \\
\hline Sex & & Louisiana & $1.4 \%$ \\
\hline Male & $48.5 \%$ & Maine & $0.4 \%$ \\
\hline Female & $51.5 \%$ & Maryland & $1.8 \%$ \\
\hline Household structure & & Massachusetts & $2.1 \%$ \\
\hline Adults only & $51.9 \%$ & Michigan & $3.0 \%$ \\
\hline Single adult & $7.8 \%$ & Minnesota & $1.7 \%$ \\
\hline Single w/ child(ren) & $2.9 \%$ & Mississippi & $0.9 \%$ \\
\hline Adults w/ child(ren) & $37.4 \%$ & Missouri & $1.8 \%$ \\
\hline Education & & Montana & $0.3 \%$ \\
\hline HS or below & $40.4 \%$ & Nebraska & $0.6 \%$ \\
\hline Some college & $30.5 \%$ & Nevada & $1.0 \%$ \\
\hline Bachelors & $16.5 \%$ & New Hampshire & $0.4 \%$ \\
\hline Graduate degree & $12.6 \%$ & New Jersey & $2.7 \%$ \\
\hline Cohort & & New Mexico & $0.6 \%$ \\
\hline 23 Apr-12 May & $9.7 \%$ & New York & $5.9 \%$ \\
\hline 14-26 May & $8.0 \%$ & North Carolina & $3.2 \%$ \\
\hline 28 May-9 Jun & $8.5 \%$ & North Dakota & $0.2 \%$ \\
\hline 11-23 Jun & $8.7 \%$ & Ohio & $3.5 \%$ \\
\hline 25 Jun-7 Jul & $8.6 \%$ & Oklahoma & $1.2 \%$ \\
\hline 9-21 Jul & $8.8 \%$ & Oregon & $1.3 \%$ \\
\hline 19-31 Aug & $6.8 \%$ & Pennsylvania & $3.9 \%$ \\
\hline 2-14 Sep & $6.8 \%$ & Rhode Island & $0.3 \%$ \\
\hline 16-28 Sep & $6.8 \%$ & South Carolina & $1.6 \%$ \\
\hline 30 Sep-12 Oct & $6.8 \%$ & South Dakota & $0.3 \%$ \\
\hline $14-26$ Oct & $6.8 \%$ & Tennessee & $2.1 \%$ \\
\hline 28 Oct-9 Nov & $6.8 \%$ & Texas & $8.6 \%$ \\
\hline \multirow[t]{2}{*}{$11-23 \mathrm{Nov}$} & $6.8 \%$ & Utah & $0.9 \%$ \\
\hline & & Vermont & $0.2 \%$ \\
\hline
\end{tabular}


${ }^{a}$ Except reports of prescription medication $(\mathrm{N}=528,615)$, counseling $(\mathrm{N}=528,586)$, and unmet need for counseling $(\mathrm{N}=528,925)$. 
Table e4. Logistic regression models with interactions between race/ethnicty and cohort predicting mental health outcomes.

\begin{tabular}{|c|c|c|c|c|}
\hline iety & Depression & $\begin{array}{l}\text { Prescription } \\
\text { Medication }\end{array}$ & $\begin{array}{l}\text { Mental health } \\
\text { Counseling }\end{array}$ & $\begin{array}{l}\text { Unmet Need for } \\
\text { Counseling }\end{array}$ \\
\hline
\end{tabular}

\begin{tabular}{|c|c|c|}
\hline 23 Apr-12 MayXWhite & Ref & Ref \\
\hline 23 Apr-12 MayXBlack & Ref & Ref \\
\hline 23 Apr-12 MayXAsian & Ref & Ref \\
\hline 23 Apr-12 MayXOther & Ref & Ref \\
\hline $\begin{array}{l}23 \text { Apr-12 } \\
\text { MayXHispanic }\end{array}$ & Ref & Ref \\
\hline 14-26 MayXWhite & Ref & Ref \\
\hline 14-26 MayXBlack & $\begin{array}{c}0.954(0.825- \\
1.105)\end{array}$ & $\begin{array}{c}0.956(0.825- \\
1.108)\end{array}$ \\
\hline 14-26 MayXAsian & $\begin{array}{c}0.897(0.722- \\
1.115)\end{array}$ & $\begin{array}{c}0.929(0.742- \\
1.163)\end{array}$ \\
\hline 14-26 MayXOther & $\begin{array}{c}0.929(0.745- \\
1.159)\end{array}$ & $\begin{array}{c}1.037(0.821- \\
1.309)\end{array}$ \\
\hline 14-26 MayXHispanic & $\begin{array}{c}0.932(0.785- \\
1.108)\end{array}$ & $\begin{array}{c}0.942(0.788- \\
1.126)\end{array}$ \\
\hline 28 May-9 JunXWhite & Ref & Ref \\
\hline 28 May-9 JunXBlack & $\begin{array}{c}0.988(0.855- \\
1.141)\end{array}$ & $\begin{array}{c}1.055(0.910- \\
1.223)\end{array}$ \\
\hline 28 May-9 JunXAsian & $\begin{array}{c}0.913(0.736- \\
1.132)\end{array}$ & $\begin{array}{c}0.861(0.690- \\
1.074)\end{array}$ \\
\hline 28 May-9 JunXOther & $\begin{array}{c}0.975(0.779- \\
1.220)\end{array}$ & $\begin{array}{c}1.009(0.792- \\
1.286)\end{array}$ \\
\hline $\begin{array}{l}28 \text { May-9 } \\
\text { JunXHispanic }\end{array}$ & $\begin{array}{c}0.779(0.667- \\
0.910)\end{array}$ & $\begin{array}{c}0.797(0.678- \\
0.936)\end{array}$ \\
\hline 11-23 JunXWhite & Ref & Ref \\
\hline 11-23 JunXBlack & $\begin{array}{c}0.922(0.794- \\
1.071)\end{array}$ & $\begin{array}{c}0.936(0.803- \\
1.091)\end{array}$ \\
\hline 11-23 JunXAsian & $\begin{array}{c}0.770(0.607- \\
0.978)\end{array}$ & $\begin{array}{c}0.810(0.631- \\
1.040)\end{array}$ \\
\hline 11-23 JunXOther & $\begin{array}{c}0.894(0.701- \\
1.140)\end{array}$ & $\begin{array}{c}1.045(0.808- \\
1.350)\end{array}$ \\
\hline 11-23 JunXHispanic & $\begin{array}{c}0.808(0.687- \\
0.950)\end{array}$ & $\begin{array}{c}0.806(0.680- \\
0.955)\end{array}$ \\
\hline 25 Jun-7 JulXWhite & Ref & Ref \\
\hline 25 Jun-7 JulXBlack & $\begin{array}{c}0.899(0.778- \\
1.039)\end{array}$ & $\begin{array}{c}0.995(0.856- \\
1.156)\end{array}$ \\
\hline 25 Jun-7 JulXAsian & $\begin{array}{c}0.741(0.594- \\
0.923)\end{array}$ & $\begin{array}{c}0.833(0.655- \\
1.060)\end{array}$ \\
\hline 25 Jun-7 JulXOther & $\begin{array}{c}0.937(0.746- \\
1.178)\end{array}$ & $\begin{array}{c}1.006(0.786- \\
1.288)\end{array}$ \\
\hline 25 Jun-7 JulXHispanic & $\begin{array}{c}0.768(0.659- \\
0.895)\end{array}$ & $\begin{array}{c}0.811(0.690- \\
0.953)\end{array}$ \\
\hline 9-21 JulXWhite & Ref & Ref \\
\hline 9-21 JulXBlack & $\begin{array}{c}0.859(0.744- \\
0.992)\end{array}$ & $\begin{array}{c}0.940(0.810- \\
1.090)\end{array}$ \\
\hline 9-21 JulXAsian & $\begin{array}{c}0.932(0.753- \\
1.155)\end{array}$ & $\begin{array}{c}0.937(0.749- \\
1.173)\end{array}$ \\
\hline 9-21 JulXOther & $0.907(0.731-$ & $0.983(0.783-$ \\
\hline
\end{tabular}


1.125)

9-21 JulXHispanic

19-31 AugXWhite

19-31 AugXBlack

19-31 AugXAsian

19-31 AugXOther

19-31 AugXOther

19-31 AugXHispanic

2-14 SepXWhite

2-14 SepXBlack

2-14 SepXAsian

2-14 SepXOther

2-14 SepXHispanic

16-28 SepXWhite

16-28 SepXBlack

16-28 SepXAsian

16-28 SepXOther

16-28 SepXHispanic

30 Sep-12 OctXWhite

30 Sep-12 OctXBlack

30 Sep-12 OctXAsian

30 Sep-12 OctXOther

30 Sep-12

OctXHispanic

14-26 OctXWhite

14-26 OctXBlack

14-26 OctXAsian

14-26 OctXOther

14-26 OctXHispanic

28 Oct-9 NovXWhite

28 Oct-9 NovXBlack

$\begin{array}{cc}0.865(0.740- & 0.863(0.733- \\ 1.011) & 1.015)\end{array}$

Ref Ref

0.947 (0.825- $\quad 1.041$ (0.905-

1.197)

1.086)

$0.851(0.695-$

1.043)

$0.791(0.636-$

$0.984)$

$0.839(0.720-$

0.978)

Ref

$1.015(0.881-$

$1.169)$

$0.748(0.614-$

0.912)

$0.959(0.774-$

1.189)

$0.911(0.783-$

1.062)

Ref

$0.940(0.814-$

1.086)

$0.803(0.650-$

$0.991)$

$0.784(0.633-$

$0.971)$

$0.908(0.774-$

1.066)

Ref

$0.962(0.830-$

1.114)

0.869 (0.706-

1.070)

$0.838(0.670-$

1.048)

0.895 (0.761-

1.053)

Ref

1.032 (0.887-

1.201)

0.981 (0.791-

1.217)

$0.912(0.720-$

1.154)

0.901 (0.764-

1.061)

Ref

0.971 (0.813-

1.159)
0.901 (0.731-

1.111)

$0.906(0.719$

1.141)

1.114)

Ref

1.111 (0.962-

1.283)

0.848 (0.691-

1.041)

0.955 (0.760-

1.200)

$0.950(0.811-$

1.112)

Ref

0.993 (0.858-

1.150)

0.847 (0.681-

1.053)

$0.847(0.675-$

1.063)

$0.945(0.801-$

1.115)

Ref

1.136 (0.979-

1.317)

$0.882(0.712-$

1.093)

$0.977(0.772-$

1.237)

1.018 (0.863-

1.202)

Ref

1.152 (0.987-

1.344)

0.981 (0.785-

1.227)

$1.009(0.790$

1.290)

1.008 (0.851-

1.193)

Ref

1.145 (0.957-

1.368)
0.949 (0.810-

Re

Ref

Ref

Ref

Ref

Ref

Ref

Ref

Ref

Ref

Ref

Ref

Ref

Ref

Ref

Ref

Ref

Ref

Ref

$1.234(1.019-$
$1.493)$

1.237 (0.970-

1.181 (0.944-1.478)

1.577)

$1.038(0.788-$

0.823 (0.584-

$0.887(0.636-1.236)$

1.158)

\begin{tabular}{ccc}
$1.003(0.771-$ & $1.126(0.784-$ & $1.136(0.856-1.507)$ \\
$1.304)$ & $1.616)$ & \\
$1.205(1.019-$ & $1.079(0.866-$ & $0.999(0.814-1.226)$ \\
$1.425)$ & $1.344)$ & \\
\hline
\end{tabular}

1.425)

Ref

Ref

\begin{tabular}{ccc}
$1.062(0.879-$ & $1.056(0.827-$ & $0.969(0.775-1.212)$ \\
$1.283)$ & $1.347)$ & \\
\hline $1.060(0.784$ & $0.834(0.598-$ & $1.175(0.846-1.632)$
\end{tabular}

$1.060(0.784-$
$1.435)$

0.834 (0.598-

$1.175(0.846-1.632)$

1.163)

$\begin{array}{lll}0.891(0.704- & 0.981(0.719- & 1.002(0.759-1.322)\end{array}$

$1.127)$

1.340)

\begin{tabular}{ccc}
$1.276(1.072-$ & $1.218(0.974-$ & $1.213(0.965-1.524)$ \\
$1.519)$ & $1.524)$ & \\
\hline
\end{tabular}

Ref

Ref

Ref

$\begin{array}{ccc}1.154(0.961- & 0.978(0.783- & 1.139(0.919-1.412) \\ 1.385) & 1.223) & \\ 0.978(0.736- & 0.771(0.553- & 1.060(0.757-1.485)\end{array}$

$0.978(0.736-$

$0.771(0.553-$ 1.074)

$\begin{array}{ccc}1.165(0.916- & 1.006(0.743- & 0.915(0.696-1.203) \\ 1.482) & 1.362) & \end{array}$

$1.193(0.995$

$1.362)$

1.431) 1.262)

$1.431)$

$1.173(0.972-$

Ref

$1.200(0.950-\quad 1.109(0.842-1.462)$

1.414)

1.516)

$1.078(0.796-$

1.029 (0.717-

$1.300(0.908-1.862)$

1.476)

$1.460)$

1.352)

$1.179(0.779-\quad 1.069(0.776-1.473)$

$1.785)$

$1.320(1.081-\quad 1.601(1.239-\quad 1.006(0.801-1.263)$

1.611) 2.067)

Ref Ref

Ref

$1.076(0.861-$

1.344)

$1.192(0.912-1.558)$

1.184 (0.906-

1.548) 


\begin{tabular}{|c|c|c|c|c|c|}
\hline 28 Oct-9 NovXAsian & $\begin{array}{c}0.951(0.725- \\
1.247)\end{array}$ & $\begin{array}{c}1.019(0.774- \\
1.341)\end{array}$ & $\begin{array}{c}1.160(0.827- \\
1.628)\end{array}$ & $\begin{array}{c}0.868(0.596- \\
1.263)\end{array}$ & $1.917(1.321-2.781)$ \\
\hline 28 Oct-9 NovXOther & $\begin{array}{c}0.827(0.643- \\
1.064)\end{array}$ & $\begin{array}{c}0.916(0.706- \\
1.188)\end{array}$ & $\begin{array}{c}1.091(0.829- \\
1.437)\end{array}$ & $\begin{array}{c}1.211(0.855- \\
1.716)\end{array}$ & $0.995(0.736-1.345)$ \\
\hline $\begin{array}{l}28 \text { Oct- } 9 \\
\text { NovXHispanic }\end{array}$ & $\begin{array}{c}1.006(0.837- \\
1.210)\end{array}$ & $\begin{array}{c}1.087(0.901- \\
1.311)\end{array}$ & $\begin{array}{c}1.205(0.953- \\
1.523)\end{array}$ & $\begin{array}{c}1.234(0.959- \\
1.588)\end{array}$ & $1.421(1.108-1.824)$ \\
\hline 11-23 NovXWhite & Ref & Ref & Ref & Ref & Ref \\
\hline 11-23 NovXBlack & $\begin{array}{c}0.981(0.833- \\
1.155)\end{array}$ & $\begin{array}{c}1.098(0.933- \\
1.291)\end{array}$ & $\begin{array}{c}1.393(1.109- \\
1.751)\end{array}$ & $\begin{array}{c}1.183(0.901- \\
1.553)\end{array}$ & $1.222(0.964-1.549)$ \\
\hline 11-23 NovXAsian & $\begin{array}{c}0.797(0.631- \\
1.007)\end{array}$ & $\begin{array}{c}0.885(0.698- \\
1.122)\end{array}$ & $\begin{array}{c}0.939(0.657- \\
1.341)\end{array}$ & $\begin{array}{c}0.984(0.614- \\
1.577)\end{array}$ & $1.139(0.782-1.660)$ \\
\hline 11-23 NovXOther & $\begin{array}{c}0.910(0.714- \\
1.160)\end{array}$ & $\begin{array}{c}1.010(0.784- \\
1.300)\end{array}$ & $\begin{array}{c}0.966(0.744- \\
1.255)\end{array}$ & $\begin{array}{c}1.116(0.816- \\
1.527)\end{array}$ & $1.107(0.810-1.511)$ \\
\hline 11-23 NovXHispanic & $\begin{array}{c}0.913(0.770- \\
1.081)\end{array}$ & $\begin{array}{c}0.911(0.766- \\
1.083)\end{array}$ & $\begin{array}{c}1.176(0.970- \\
1.426)\end{array}$ & $\begin{array}{c}1.247(0.976- \\
1.592)\end{array}$ & $1.193(0.941-1.512)$ \\
\hline Wald p-value & 0.005 & 0.004 & 0.216 & 0.176 & 0.012 \\
\hline Observations & $1,483,378$ & $1,483,378$ & 528,615 & 528,586 & 528,925 \\
\hline
\end{tabular}

Note. OR Odds Ratio. Cl Confidence Intervals. Models also adjust for race/ethnicity, age, sex, household structure, education, cohort, and state of residence.

ORs and Cls in italics significant at $p<.01$. 


\begin{tabular}{|c|c|c|c|c|c|}
\hline & Anxiety & Depression & $\begin{array}{l}\text { Prescription } \\
\text { Medication }\end{array}$ & $\begin{array}{l}\text { Mental health } \\
\text { Counseling }\end{array}$ & $\begin{array}{c}\text { Unmet Need for } \\
\text { Counseling }\end{array}$ \\
\hline $\begin{array}{l}23 \text { Apr-12 MayX18- } \\
29\end{array}$ & Ref & Ref & & & \\
\hline $\begin{array}{l}23 \text { Apr-12 MayX30- } \\
39\end{array}$ & Ref & Ref & & & \\
\hline $\begin{array}{l}23 \text { Apr-12 MayX40- } \\
49\end{array}$ & Ref & Ref & & & \\
\hline $\begin{array}{l}23 \text { Apr-12 MayX50- } \\
59\end{array}$ & Ref & Ref & & & \\
\hline $\begin{array}{l}23 \text { Apr-12 MayX60- } \\
69\end{array}$ & Ref & Ref & & & \\
\hline 23 Apr-12 MayX70+ & Ref & Ref & & & \\
\hline 14-26 MayX18-29 & Ref & Ref & & & \\
\hline 14-26 МayX30-39 & $\begin{array}{c}0.854(0.726- \\
1.004)\end{array}$ & $\begin{array}{c}0.714(0.604- \\
0.843)\end{array}$ & & & \\
\hline 14-26 MayX40-49 & $\begin{array}{c}0.795(0.673- \\
0.940)\end{array}$ & $\begin{array}{c}0.689(0.579- \\
0.819)\end{array}$ & & & \\
\hline 14-26 MayX50-59 & $\begin{array}{c}0.816(0.684- \\
0.974)\end{array}$ & $\begin{array}{c}0.767(0.638- \\
0.923)\end{array}$ & & & \\
\hline 14-26 MayX60-69 & $\begin{array}{c}0.902(0.755- \\
1.076)\end{array}$ & $\begin{array}{c}0.853(0.711- \\
1.022)\end{array}$ & & & \\
\hline 14-26 MayX70+ & $\begin{array}{c}0.925(0.749- \\
1.142)\end{array}$ & $\begin{array}{c}0.923(0.742- \\
1.148)\end{array}$ & & & \\
\hline $\begin{array}{l}28 \text { May-9 JunX18- } \\
29\end{array}$ & Ref & Ref & & & \\
\hline $\begin{array}{l}28 \text { May-9 JunX30- } \\
39\end{array}$ & $\begin{array}{c}0.853(0.734- \\
0.992)\end{array}$ & $\begin{array}{c}0.786(0.673- \\
0.919)\end{array}$ & & & \\
\hline $\begin{array}{l}28 \text { May-9 JunX40- } \\
49\end{array}$ & $\begin{array}{c}0.783(0.669- \\
0.917)\end{array}$ & $\begin{array}{c}0.792(0.673- \\
0.932)\end{array}$ & & & \\
\hline $\begin{array}{l}28 \text { May-9 JunX50- } \\
59\end{array}$ & $\begin{array}{c}0.771(0.654- \\
0.910)\end{array}$ & $\begin{array}{c}0.781(0.657- \\
0.927)\end{array}$ & & & \\
\hline $\begin{array}{l}28 \text { May-9 JunX60- } \\
69\end{array}$ & $\begin{array}{c}0.794(0.671- \\
0.940)\end{array}$ & $\begin{array}{l}0.795(0.669- \\
0.945)\end{array}$ & & & \\
\hline 28 May-9 JunX70+ & $\begin{array}{c}0.923(0.765- \\
1.113)\end{array}$ & $\begin{array}{c}0.906(0.744- \\
1.103)\end{array}$ & & & \\
\hline 11-23 JunX18-29 & Ref & Ref & & & \\
\hline 11-23 JunX30-39 & $\begin{array}{c}0.864(0.735- \\
1.015)\end{array}$ & $\begin{array}{c}0.753(0.638- \\
0.890)\end{array}$ & & & \\
\hline 11-23 JunX40-49 & $\begin{array}{c}0.881(0.747- \\
1.038)\end{array}$ & $\begin{array}{c}0.841(0.709- \\
0.997)\end{array}$ & & & \\
\hline 11-23 JunX50-59 & $\begin{array}{c}0.842(0.709- \\
1.000)\end{array}$ & $\begin{array}{c}0.813(0.679- \\
0.974)\end{array}$ & & & \\
\hline 11-23 JunX60-69 & $\begin{array}{c}0.841(0.705- \\
1.004)\end{array}$ & $\begin{array}{c}0.871(0.724- \\
1.047)\end{array}$ & & & \\
\hline 11-23 JunX70+ & $\begin{array}{c}0.918(0.746- \\
1.129)\end{array}$ & $\begin{array}{c}0.949(0.764- \\
1.179)\end{array}$ & & & \\
\hline 25 Jun-7 JulX18-29 & Ref & Ref & & & \\
\hline 25 Jun-7 JulX30-39 & $\begin{array}{c}0.965(0.829- \\
1.124)\end{array}$ & $\begin{array}{c}0.917(0.784- \\
1.074)\end{array}$ & & & \\
\hline
\end{tabular}




\begin{tabular}{|c|c|c|c|c|c|}
\hline 25 Jun-7 JulX40-49 & $\begin{array}{c}0.877(0.749- \\
1.027)\end{array}$ & $\begin{array}{c}0.884(0.751- \\
1.041)\end{array}$ & & & \\
\hline 25 Jun-7 JulX50-59 & $\begin{array}{c}0.855(0.726- \\
1.007)\end{array}$ & $\begin{array}{c}0.898(0.756- \\
1.067)\end{array}$ & & & \\
\hline 25 Jun-7 JulX60-69 & $\begin{array}{c}0.927(0.784- \\
1.096)\end{array}$ & $\begin{array}{c}1.024(0.860- \\
1.220)\end{array}$ & & & \\
\hline 25 Jun-7 JulX70+ & $\begin{array}{c}0.841(0.696- \\
1.016)\end{array}$ & $\begin{array}{c}0.966(0.790- \\
1.182)\end{array}$ & & & \\
\hline 9-21 JulX18-29 & Ref & Ref & & & \\
\hline 9-21 JulX30-39 & $\begin{array}{c}0.933(0.800- \\
1.089)\end{array}$ & $\begin{array}{c}0.873(0.744- \\
1.024)\end{array}$ & & & \\
\hline 9-21 JulX40-49 & $\begin{array}{c}0.834(0.713- \\
0.976)\end{array}$ & $\begin{array}{c}0.890(0.758- \\
1.046)\end{array}$ & & & \\
\hline 9-21 JulX50-59 & $\begin{array}{c}0.787(0.669- \\
0.927)\end{array}$ & $\begin{array}{c}0.828(0.699- \\
0.982)\end{array}$ & & & \\
\hline 9-21 JulX60-69 & $\begin{array}{c}0.833(0.706- \\
0.983)\end{array}$ & $\begin{array}{c}0.940(0.791- \\
1.116)\end{array}$ & & & \\
\hline 9-21 JulX70+ & $\begin{array}{c}0.847(0.706- \\
1.017)\end{array}$ & $\begin{array}{c}0.910(0.751- \\
1.103)\end{array}$ & & & \\
\hline 19-31 AugX18-29 & Ref & Ref & Ref & Ref & Ref \\
\hline 19-31 AugX30-39 & $\begin{array}{c}0.881(0.758- \\
1.023)\end{array}$ & $\begin{array}{c}0.805(0.691- \\
0.939)\end{array}$ & Ref & Ref & Ref \\
\hline 19-31 AugX40-49 & $\begin{array}{c}0.796(0.682- \\
0.929)\end{array}$ & $\begin{array}{c}0.780(0.666- \\
0.914)\end{array}$ & Ref & Ref & Ref \\
\hline 19-31 AugX50-59 & $\begin{array}{c}0.778(0.664- \\
0.912)\end{array}$ & $\begin{array}{c}0.765(0.648- \\
0.903)\end{array}$ & Ref & Ref & Ref \\
\hline 19-31 AugX60-69 & $\begin{array}{c}0.837(0.713- \\
0.983)\end{array}$ & $\begin{array}{c}0.846(0.716- \\
0.998)\end{array}$ & Ref & Ref & Ref \\
\hline 19-31 AugX70+ & $\begin{array}{c}0.840(0.702- \\
1.006)\end{array}$ & $\begin{array}{c}0.824(0.682- \\
0.995)\end{array}$ & Ref & Ref & Ref \\
\hline 2-14 SepX18-29 & Ref & Ref & Ref & Ref & Ref \\
\hline 2-14 SepX30-39 & $\begin{array}{c}0.919(0.789- \\
1.070)\end{array}$ & $\begin{array}{c}0.814(0.697- \\
0.952)\end{array}$ & $\begin{array}{c}1.119(0.940- \\
1.332)\end{array}$ & $\begin{array}{c}1.003(0.818- \\
1.230)\end{array}$ & $1.072(0.886-1.298)$ \\
\hline 2-14 SepX40-49 & $\begin{array}{c}0.806(0.689- \\
0.942)\end{array}$ & $\begin{array}{c}0.819(0.698- \\
0.961)\end{array}$ & $\begin{array}{c}1.011(0.851- \\
1.201)\end{array}$ & $\begin{array}{c}1.008(0.814- \\
1.248)\end{array}$ & $1.128(0.926-1.374)$ \\
\hline 2-14 SepX50-59 & $\begin{array}{c}0.777(0.662- \\
0.913)\end{array}$ & $\begin{array}{c}0.744(0.630- \\
0.880)\end{array}$ & $\begin{array}{c}1.017(0.856- \\
1.210)\end{array}$ & $\begin{array}{c}0.896(0.715- \\
1.123)\end{array}$ & $0.974(0.790-1.201)$ \\
\hline 2-14 SepX60-69 & $\begin{array}{c}0.830(0.705- \\
0.977)\end{array}$ & $\begin{array}{c}0.838(0.708- \\
0.991)\end{array}$ & $\begin{array}{c}1.122(0.943- \\
1.336)\end{array}$ & $\begin{array}{c}1.026(0.813- \\
1.296)\end{array}$ & $0.983(0.776-1.244)$ \\
\hline 2-14 SepX70+ & $\begin{array}{c}0.883(0.736- \\
1.058)\end{array}$ & $\begin{array}{c}0.824(0.682- \\
0.996)\end{array}$ & $\begin{array}{c}0.989(0.810- \\
1.209)\end{array}$ & $\begin{array}{c}0.882(0.662- \\
1.174)\end{array}$ & $1.283(0.933-1.763)$ \\
\hline 16-28 SepX18-29 & Ref & Ref & Ref & Ref & Ref \\
\hline 16-28 SepX30-39 & $\begin{array}{c}0.941(0.804- \\
1.102)\end{array}$ & $\begin{array}{c}0.859(0.731- \\
1.009)\end{array}$ & $\begin{array}{c}1.110(0.930- \\
1.324)\end{array}$ & $\begin{array}{c}1.129(0.921- \\
1.386)\end{array}$ & $0.946(0.773-1.158)$ \\
\hline 16-28 SepX40-49 & $\begin{array}{c}0.820(0.697- \\
0.964)\end{array}$ & $\begin{array}{c}0.808(0.684- \\
0.954)\end{array}$ & $\begin{array}{c}0.991(0.833- \\
1.180)\end{array}$ & $\begin{array}{c}1.071(0.866- \\
1.325)\end{array}$ & $0.898(0.724-1.113)$ \\
\hline 16-28 SepX50-59 & $\begin{array}{c}0.756(0.641- \\
0.893)\end{array}$ & $\begin{array}{c}0.745(0.627- \\
0.884)\end{array}$ & $\begin{array}{c}0.994(0.834- \\
1.183)\end{array}$ & $\begin{array}{c}0.958(0.769- \\
1.195)\end{array}$ & $0.913(0.730-1.142)$ \\
\hline 16-28 SepX60-69 & $\begin{array}{c}0.853(0.721- \\
1.010)\end{array}$ & $\begin{array}{c}0.884(0.743- \\
1.052)\end{array}$ & $\begin{array}{c}1.058(0.885- \\
1.266)\end{array}$ & $\begin{array}{c}1.159(0.906- \\
1.483)\end{array}$ & $0.875(0.686-1.115)$ \\
\hline 16-28 SepX70+ & $0.802(0.666-$ & $0.780(0.643-$ & 0.877 (0.719- & $0.965(0.722-$ & $1.064(0.758-1.494)$ \\
\hline
\end{tabular}




\begin{tabular}{|c|c|c|c|c|c|}
\hline & $0.965)$ & $0.946)$ & $1.070)$ & 1.291) & \\
\hline $\begin{array}{l}30 \text { Sep-12 OctX18- } \\
29\end{array}$ & Ref & Ref & Ref & Ref & Ref \\
\hline $\begin{array}{l}30 \text { Sep-12 OctX30- } \\
39\end{array}$ & $\begin{array}{c}0.864(0.735- \\
1.016)\end{array}$ & $\begin{array}{c}0.791(0.671- \\
0.931)\end{array}$ & $\begin{array}{c}1.149(0.962- \\
1.372)\end{array}$ & $\begin{array}{c}1.014(0.826- \\
1.244)\end{array}$ & $1.013(0.833-1.233)$ \\
\hline $\begin{array}{l}30 \text { Sep-12 OctX40- } \\
49\end{array}$ & $\begin{array}{c}0.795(0.673- \\
0.939)\end{array}$ & $\begin{array}{c}0.789(0.667- \\
0.934)\end{array}$ & $\begin{array}{c}1.136(0.951- \\
1.356)\end{array}$ & $\begin{array}{c}1.067(0.859- \\
1.325)\end{array}$ & $0.998(0.821-1.214)$ \\
\hline $\begin{array}{l}30 \text { Sep-12 OctX50- } \\
59\end{array}$ & $\begin{array}{c}0.730(0.616- \\
0.865)\end{array}$ & $\begin{array}{c}0.683(0.574- \\
0.813)\end{array}$ & $\begin{array}{c}1.088(0.908- \\
1.304)\end{array}$ & $\begin{array}{c}0.950(0.756- \\
1.192)\end{array}$ & $1.009(0.814-1.249)$ \\
\hline $\begin{array}{l}30 \text { Sep-12 OctX60- } \\
69\end{array}$ & $\begin{array}{c}0.746(0.628- \\
0.885)\end{array}$ & $\begin{array}{l}0.757(0.635- \\
0.902)\end{array}$ & $\begin{array}{c}1.109(0.926- \\
1.327)\end{array}$ & $\begin{array}{c}0.988(0.783- \\
1.249)\end{array}$ & $0.897(0.707-1.139)$ \\
\hline 30 Sep-12 OctX70+ & $\begin{array}{c}0.757(0.627- \\
0.914)\end{array}$ & $\begin{array}{c}0.719(0.591- \\
0.874)\end{array}$ & $\begin{array}{c}1.075(0.874- \\
1.324)\end{array}$ & $\begin{array}{c}0.988(0.727- \\
1.341)\end{array}$ & 0.908 (0.656-1.259) \\
\hline 14-26 OctX18-29 & Ref & Ref & Ref & Ref & Ref \\
\hline 14-26 OctX30-39 & $\begin{array}{l}0.957(0.813- \\
1.127)\end{array}$ & $\begin{array}{c}0.854(0.724- \\
1.008)\end{array}$ & $\begin{array}{c}1.155(0.948- \\
1.408)\end{array}$ & $\begin{array}{c}1.056(0.842- \\
1.324)\end{array}$ & $1.004(0.812-1.242)$ \\
\hline 14-26 OctX40-49 & $\begin{array}{c}0.838(0.708- \\
0.993)\end{array}$ & $\begin{array}{c}0.819(0.690- \\
0.972)\end{array}$ & $\begin{array}{c}1.039(0.856- \\
1.261)\end{array}$ & $\begin{array}{c}0.914(0.727- \\
1.150)\end{array}$ & $0.910(0.732-1.131)$ \\
\hline 14-26 OctX50-59 & $\begin{array}{c}0.722(0.606- \\
0.859)\end{array}$ & $\begin{array}{c}0.707(0.591- \\
0.846)\end{array}$ & $\begin{array}{c}1.090(0.893- \\
1.330)\end{array}$ & $\begin{array}{c}0.959(0.747- \\
1.230)\end{array}$ & $0.957(0.738-1.241)$ \\
\hline 14-26 OctX60-69 & $\begin{array}{c}0.825(0.694- \\
0.981)\end{array}$ & $\begin{array}{c}0.804(0.673- \\
0.959)\end{array}$ & $\begin{array}{c}0.958(0.790- \\
1.162)\end{array}$ & $\begin{array}{c}0.901(0.704- \\
1.154)\end{array}$ & $0.835(0.651-1.071)$ \\
\hline 14-26 OctX70+ & $\begin{array}{c}0.790(0.650- \\
0.961)\end{array}$ & $\begin{array}{c}0.745(0.609- \\
0.913)\end{array}$ & $\begin{array}{c}0.914(0.726- \\
1.151)\end{array}$ & $\begin{array}{c}0.824(0.532- \\
1.276)\end{array}$ & $1.151(0.785-1.688)$ \\
\hline 28 Oct-9 NovX18-29 & Ref & Ref & Ref & Ref & Ref \\
\hline 28 Oct-9 NovX30-39 & $\begin{array}{c}0.809(0.667- \\
0.982)\end{array}$ & $\begin{array}{c}0.825(0.681- \\
1.000)\end{array}$ & $\begin{array}{c}1.121(0.890- \\
1.412)\end{array}$ & $\begin{array}{c}1.013(0.793- \\
1.293)\end{array}$ & $0.879(0.694-1.112)$ \\
\hline 28 Oct-9 NovX40-49 & $\begin{array}{c}0.713(0.588- \\
0.865)\end{array}$ & $\begin{array}{c}0.722(0.595- \\
0.876)\end{array}$ & $\begin{array}{c}0.883(0.709- \\
1.099)\end{array}$ & $\begin{array}{c}0.822(0.640- \\
1.056)\end{array}$ & $0.760(0.602-0.960)$ \\
\hline 28 Oct-9 NovX50-59 & $\begin{array}{c}0.620(0.509- \\
0.756)\end{array}$ & $\begin{array}{c}0.642(0.526- \\
0.784)\end{array}$ & $\begin{array}{c}0.903(0.724- \\
1.127)\end{array}$ & $\begin{array}{c}0.897(0.681- \\
1.181)\end{array}$ & $0.883(0.681-1.144)$ \\
\hline 28 Oct- 9 NovX60-69 & $\begin{array}{c}0.634(0.520- \\
0.774)\end{array}$ & $\begin{array}{c}0.636(0.520- \\
0.778)\end{array}$ & $\begin{array}{c}0.989(0.790- \\
1.236)\end{array}$ & $\begin{array}{c}0.928(0.699- \\
1.230)\end{array}$ & $0.706(0.533-0.934)$ \\
\hline 28 Oct-9 NovX70+ & $\begin{array}{c}0.664(0.533- \\
0.826)\end{array}$ & $\begin{array}{c}0.663(0.530- \\
0.831)\end{array}$ & $\begin{array}{c}0.886(0.690- \\
1.138)\end{array}$ & $\begin{array}{c}0.643(0.466- \\
0.887)\end{array}$ & $0.986(0.656-1.480)$ \\
\hline 11-23 NovX18-29 & Ref & Ref & Ref & Ref & Ref \\
\hline 11-23 NovX30-39 & $\begin{array}{c}0.879(0.737- \\
1.047)\end{array}$ & $\begin{array}{c}0.769(0.645- \\
0.918)\end{array}$ & $\begin{array}{c}1.178(0.965- \\
1.439)\end{array}$ & $\begin{array}{c}0.937(0.741- \\
1.184)\end{array}$ & $1.006(0.814-1.243)$ \\
\hline 11-23 NovX40-49 & $\begin{array}{c}0.833(0.696- \\
0.996)\end{array}$ & $\begin{array}{c}0.766(0.639- \\
0.918)\end{array}$ & $\begin{array}{c}1.024(0.843- \\
1.244)\end{array}$ & $\begin{array}{c}0.948(0.750- \\
1.200)\end{array}$ & $0.985(0.788-1.230)$ \\
\hline 11-23 NovX50-59 & $\begin{array}{c}0.738(0.614- \\
0.887)\end{array}$ & $\begin{array}{c}0.700(0.580- \\
0.845)\end{array}$ & $\begin{array}{c}1.146(0.943- \\
1.394)\end{array}$ & $\begin{array}{c}0.833(0.651- \\
1.066)\end{array}$ & $1.175(0.923-1.495)$ \\
\hline 11-23 NovX60-69 & $\begin{array}{c}0.756(0.630- \\
0.909)\end{array}$ & $\begin{array}{c}0.675(0.560- \\
0.814)\end{array}$ & $\begin{array}{c}1.093(0.899- \\
1.329)\end{array}$ & $\begin{array}{c}0.886(0.677- \\
1.160)\end{array}$ & $0.964(0.745-1.248)$ \\
\hline 11-23 NovX70+ & $\begin{array}{c}0.833(0.680- \\
1.020) \\
\end{array}$ & $\begin{array}{c}0.750(0.609- \\
0.923)\end{array}$ & $\begin{array}{c}0.984(0.783- \\
1.238)\end{array}$ & $\begin{array}{c}0.827(0.597- \\
1.146)\end{array}$ & $1.070(0.734-1.562)$ \\
\hline Wald p-value & 0.002 & 0.000 & 0.194 & 0.415 & 0.421 \\
\hline Observations & $1,483,378$ & $1,483,378$ & 528,615 & 528,586 & 528,925 \\
\hline
\end{tabular}

Note. OR Odds Ratio. CI Confidence Intervals. Models also adjust for race/ethnicity, age, sex, household structure, education, cohort, and state of residence. 
ORs and $\mathrm{Cls}$ in italics significant at $p<.01$.

Table e6. Logistic regression models with interactions between sex and cohort predicting mental health outcomes.

\begin{tabular}{|c|c|c|c|c|c|}
\hline & Anxiety & Depression & $\begin{array}{l}\text { Prescription } \\
\text { Medication }\end{array}$ & $\begin{array}{l}\text { Mental health } \\
\text { Counseling }\end{array}$ & $\begin{array}{c}\text { Unmet Need for } \\
\text { Counseling }\end{array}$ \\
\hline 23 Apr-12 MayXMale & Ref & Ref & & & \\
\hline 23 Apr-12 & Ref & Ref & & & \\
\hline \multicolumn{6}{|l|}{ MayXFemale } \\
\hline 14-26 MayXMale & Ref & Ref & & & \\
\hline 14-26 MayXFemale & $\begin{array}{c}0.912(0.826- \\
1.008)\end{array}$ & $\begin{array}{c}0.919(0.827- \\
1.020)\end{array}$ & & & \\
\hline 28 May-9 JunXMale & Ref & Ref & & & \\
\hline 28 May-9 & $0.983(0.896-$ & $0.928(0.842-$ & & & \\
\hline JunXFemale & $1.078)$ & $1.023)$ & & & \\
\hline 11-23 JunXMale & Ref & Ref & & & \\
\hline 11-23 JunXFemale & $\begin{array}{c}0.900(0.816- \\
0.993)\end{array}$ & $\begin{array}{c}0.901(0.811- \\
1.000)\end{array}$ & & & \\
\hline 25 Jun-7 JulXMale & Ref & Ref & & & \\
\hline 25 Jun-7 JulXFemale & $\begin{array}{c}0.961(0.876- \\
1.054)\end{array}$ & $\begin{array}{c}0.907(0.821- \\
1.001)\end{array}$ & & & \\
\hline 9-21 JulXMale & Ref & Ref & & & \\
\hline 9-21 JulXFemale & $\begin{array}{c}0.938(0.856- \\
1.029)\end{array}$ & $\begin{array}{c}0.914(0.829- \\
1.008)\end{array}$ & & & \\
\hline 19-31 AugXMale & Ref & Ref & Ref & Ref & Ref \\
\hline 19-31 AugXFemale & $\begin{array}{c}0.957(0.877- \\
1.046)\end{array}$ & $\begin{array}{c}0.894(0.814- \\
0.981)\end{array}$ & Ref & Ref & Ref \\
\hline 2-14 SepXMale & Ref & Ref & Ref & Ref & Ref \\
\hline 2-14 SepXFemale & $\begin{array}{c}0.902(0.825- \\
0.985)\end{array}$ & $\begin{array}{c}0.857(0.780- \\
0.941)\end{array}$ & $\begin{array}{c}0.882(0.799- \\
0.972)\end{array}$ & $\begin{array}{c}0.998(0.872- \\
1.142)\end{array}$ & $1.080(0.940-1.241)$ \\
\hline 16-28 SepXMale & Ref & Ref & Ref & Ref & Ref \\
\hline 16-28 SepXFemale & $\begin{array}{c}0.948(0.866- \\
1.039)\end{array}$ & $\begin{array}{c}0.916(0.832- \\
1.008)\end{array}$ & $\begin{array}{c}0.949(0.861- \\
1.047)\end{array}$ & $\begin{array}{c}1.005(0.877- \\
1.151)\end{array}$ & $1.014(0.875-1.176)$ \\
\hline 30 Sep-12 OctXMale & Ref & Ref & Ref & Ref & Ref \\
\hline 30 Sep-12 & $0.916(0.835-$ & $0.895(0.812-$ & $0.885(0.801-$ & 0.899 (0.786- & $1.040(0.903-1.197)$ \\
\hline OctXFemale & 1.004) & 0.986) & $0.977)$ & $1.028)$ & \\
\hline 14-26 OctXMale & Ref & Ref & Ref & Ref & Ref \\
\hline 14-26 OctXFemale & $\begin{array}{c}0.948(0.863- \\
1.042)\end{array}$ & $\begin{array}{c}0.892(0.808- \\
0.984)\end{array}$ & $\begin{array}{c}0.888(0.797- \\
0.989)\end{array}$ & $\begin{array}{c}0.887(0.762- \\
1.032)\end{array}$ & $0.906(0.774-1.060)$ \\
\hline 28 Oct-9 NovXMale & Ref & Ref & Ref & Ref & Ref \\
\hline 28 Oct-9 NovXFemale & $\begin{array}{c}0.942(0.847- \\
1.046)\end{array}$ & $\begin{array}{c}0.837(0.750- \\
0.935)\end{array}$ & $\begin{array}{c}0.944(0.835- \\
1.066)\end{array}$ & $\begin{array}{c}0.968(0.827- \\
1.134)\end{array}$ & $0.833(0.707-0.980)$ \\
\hline 11-23 NovXMale & Ref & Ref & Ref & Ref & Ref \\
\hline 11-23 NovXFemale & $\begin{array}{c}1.004(0.910- \\
1.108)\end{array}$ & $\begin{array}{c}0.928(0.837- \\
1.028)\end{array}$ & $\begin{array}{c}0.925(0.826- \\
1.035)\end{array}$ & $\begin{array}{c}0.863(0.740- \\
1.007)\end{array}$ & $1.015(0.868-1.187)$ \\
\hline Wald p-value & 0.252 & 0.181 & 0.132 & 0.207 & 0.049 \\
\hline Observations & $1,483,378$ & $1,483,378$ & 528,615 & 528,586 & 528,925 \\
\hline
\end{tabular}

Note. OR Odds Ratio. Cl Confidence Intervals. Models also adjust for race/ethnicity, age, sex, household structure, education, cohort, and state of residence. ORs and $\mathrm{Cls}$ in italics significant at $p<.01$. 
Page 23/28 
Table e7. Logistic regression models with interactions between household structure and cohort predicting mental health outcomes.

Anxiety Depression Prescription Mental health Unmet Need for Medication Counseling Counseling

\begin{tabular}{|c|c|c|c|c|c|}
\hline 23 Apr-12 MayXSingle adult & Ref & Ref & & & \\
\hline 23 Apr-12 MayXAdults only & Ref & Ref & & & \\
\hline $\begin{array}{l}23 \text { Apr-12 MayXSingle w/ } \\
\text { child(ren) }\end{array}$ & Ref & Ref & & & \\
\hline $\begin{array}{l}23 \text { Apr-12 MayXAdults } \\
\text { w/child(ren) }\end{array}$ & Ref & Ref & & & \\
\hline 14-26 MayXSingle adult & $\begin{array}{c}1.076(0.942- \\
1.230)\end{array}$ & $\begin{array}{c}1.095(0.953- \\
1.258)\end{array}$ & & & \\
\hline 14-26 MayXAdults only & Ref & Ref & & & \\
\hline $\begin{array}{l}\text { 14-26 MayXSingle w/ } \\
\text { child(ren) }\end{array}$ & $\begin{array}{c}1.169(0.953- \\
1.433)\end{array}$ & $\begin{array}{c}1.089(0.888- \\
1.337)\end{array}$ & & & \\
\hline $\begin{array}{l}\text { 14-26 MayXAdults } \\
\text { w/child(ren) }\end{array}$ & $\begin{array}{c}1.029(0.923- \\
1.148)\end{array}$ & $\begin{array}{c}1.046(0.932- \\
1.174)\end{array}$ & & & \\
\hline 28 May-9 JunXSingle adult & $\begin{array}{c}1.080(0.944- \\
1.236)\end{array}$ & $\begin{array}{c}1.067(0.928- \\
1.227)\end{array}$ & & & \\
\hline 28 May-9 JunXAdults only & Ref & Ref & & & \\
\hline $\begin{array}{l}28 \text { May-9 JunXSingle w/ } \\
\text { child(ren) }\end{array}$ & $\begin{array}{c}1.201(0.998- \\
1.445)\end{array}$ & $\begin{array}{c}1.152(0.953- \\
1.391)\end{array}$ & & & \\
\hline $\begin{array}{l}28 \text { May-9 JunXAdults } \\
\text { w/child(ren) }\end{array}$ & $\begin{array}{c}0.997(0.901- \\
1.103)\end{array}$ & $\begin{array}{c}1.005(0.902- \\
1.119)\end{array}$ & & & \\
\hline 11-23 JunXSingle adult & $\begin{array}{c}0.964(0.841- \\
1.105)\end{array}$ & $\begin{array}{c}0.967(0.840- \\
1.114)\end{array}$ & & & \\
\hline 11-23 JunXAdults only & Ref & Ref & & & \\
\hline $\begin{array}{l}\text { 11-23 JunXSingle w/ } \\
\text { child(ren) }\end{array}$ & $\begin{array}{l}0.970(0.800- \\
1.176)\end{array}$ & $\begin{array}{c}0.965(0.787- \\
1.185)\end{array}$ & & & \\
\hline 11-23 JunXAdults w/child(ren) & $\begin{array}{c}0.969(0.871- \\
1.079)\end{array}$ & $\begin{array}{c}0.919(0.819- \\
1.031)\end{array}$ & & & \\
\hline 25 Jun-7 JulXSingle adult & $\begin{array}{c}1.003(0.880- \\
1.144)\end{array}$ & $\begin{array}{c}1.055(0.920- \\
1.211)\end{array}$ & & & \\
\hline 25 Jun-7 JulXAdults only & Ref & Ref & & & \\
\hline $\begin{array}{l}25 \text { Jun-7 JulXSingle w/ } \\
\text { child(ren) }\end{array}$ & $\begin{array}{c}1.092(0.920- \\
1.295)\end{array}$ & $\begin{array}{c}1.045(0.876- \\
1.246)\end{array}$ & & & \\
\hline $\begin{array}{l}\text { 25 Jun-7 JulXAdults } \\
\text { w/child(ren) }\end{array}$ & $\begin{array}{c}1.057(0.955- \\
1.169)\end{array}$ & $\begin{array}{c}1.030(0.924- \\
1.149)\end{array}$ & & & \\
\hline 9-21 JulXSingle adult & $\begin{array}{c}0.968(0.855- \\
1.097)\end{array}$ & $\begin{array}{c}1.011(0.887- \\
1.153)\end{array}$ & & & \\
\hline 9-21 JulXAdults only & Ref & Ref & & & \\
\hline 9-21 JulXSingle w/ child(ren) & $\begin{array}{c}0.872(0.720- \\
1.056)\end{array}$ & $\begin{array}{c}0.971(0.796- \\
1.184)\end{array}$ & & & \\
\hline 9-21 JulXAdults w/child(ren) & $\begin{array}{c}1.013(0.917- \\
1.120)\end{array}$ & $\begin{array}{c}1.033(0.928- \\
1.150)\end{array}$ & & & \\
\hline 19-31 AugXSingle adult & $\begin{array}{c}1.008(0.899- \\
1.130)\end{array}$ & $\begin{array}{c}0.995(0.883- \\
1.121)\end{array}$ & Ref & Ref & Ref \\
\hline 19-31 AugXAdults only & Ref & Ref & Ref & Ref & Ref \\
\hline $\begin{array}{l}\text { 19-31 AugXSingle w/ } \\
\text { child(ren) }\end{array}$ & $\begin{array}{c}1.218(1.025- \\
1.447)\end{array}$ & $\begin{array}{c}1.219(1.022- \\
1.454)\end{array}$ & Ref & Ref & Ref \\
\hline 19-31 AugXAdults w/child(ren) & $\begin{array}{c}1.019(0.925- \\
1.124)\end{array}$ & $\begin{array}{c}1.047(0.944- \\
1.162)\end{array}$ & Ref & Ref & Ref \\
\hline
\end{tabular}




\begin{tabular}{|c|c|c|c|c|c|}
\hline 2-14 SepXSingle adult & $\begin{array}{l}0.997(0.888- \\
1.118)\end{array}$ & $\begin{array}{c}0.976(0.866- \\
1.101)\end{array}$ & $\begin{array}{l}0.887(0.791- \\
0.994)\end{array}$ & $\begin{array}{c}0.889(0.762- \\
1.036)\end{array}$ & $0.924(0.785-1.087)$ \\
\hline 2-14 SepXAdults only & Ref & Ref & Ref & Ref & Ref \\
\hline 2-14 SepXSingle w/ child(ren) & $\begin{array}{c}1.261(1.066- \\
1.492)\end{array}$ & $\begin{array}{c}1.088(0.916- \\
1.293)\end{array}$ & $\begin{array}{c}0.894(0.743- \\
1.074)\end{array}$ & $\begin{array}{c}0.835(0.662- \\
1.052)\end{array}$ & $0.946(0.732-1.221)$ \\
\hline 2-14 SepXAdults w/child(ren) & $\begin{array}{c}1.000(0.906- \\
1.104)\end{array}$ & $\begin{array}{c}0.987(0.889- \\
1.096)\end{array}$ & $\begin{array}{c}0.961(0.864- \\
1.068)\end{array}$ & $\begin{array}{c}0.890(0.769- \\
1.030)\end{array}$ & $0.971(0.842-1.120)$ \\
\hline 16-28 SepXSingle adult & $\begin{array}{c}1.026(0.911- \\
1.156)\end{array}$ & $\begin{array}{c}1.006(0.888- \\
1.139)\end{array}$ & $\begin{array}{l}1.041(0.925- \\
1.171)\end{array}$ & $\begin{array}{c}1.074(0.916- \\
1.260)\end{array}$ & $0.900(0.751-1.079)$ \\
\hline 16-28 SepXAdults only & Ref & Ref & Ref & Ref & Ref \\
\hline $\begin{array}{l}\text { 16-28 SepXSingle w/ } \\
\text { child(ren) }\end{array}$ & $\begin{array}{c}1.300(1.084- \\
1.561)\end{array}$ & $\begin{array}{c}1.161(0.964- \\
1.399)\end{array}$ & $\begin{array}{l}0.944(0.770- \\
1.156)\end{array}$ & $\begin{array}{c}0.944(0.729- \\
1.221)\end{array}$ & $0.825(0.633-1.076)$ \\
\hline 16-28 SepXAdults w/child(ren) & $\begin{array}{c}1.049(0.949- \\
1.160)\end{array}$ & $\begin{array}{c}1.065(0.957- \\
1.185)\end{array}$ & $\begin{array}{c}1.054(0.948- \\
1.171)\end{array}$ & $\begin{array}{c}0.975(0.843- \\
1.127)\end{array}$ & $0.992(0.856-1.150)$ \\
\hline 30 Sep-12 OctXSingle adult & $\begin{array}{c}1.098(0.974- \\
1.239)\end{array}$ & $\begin{array}{c}1.046(0.922- \\
1.186)\end{array}$ & $\begin{array}{c}1.033(0.917- \\
1.164)\end{array}$ & $\begin{array}{c}1.054(0.886- \\
1.253)\end{array}$ & $0.972(0.824-1.147)$ \\
\hline 30 Sep-12 OctXAdults only & Ref & Ref & Ref & Ref & Ref \\
\hline $\begin{array}{l}30 \text { Sep-12 OctXSingle w/ } \\
\text { child(ren) }\end{array}$ & $\begin{array}{l}1.205(1.013- \\
1.434)\end{array}$ & $\begin{array}{l}1.108(0.929- \\
1.321)\end{array}$ & $\begin{array}{l}0.991(0.816- \\
1.203)\end{array}$ & $\begin{array}{c}0.809(0.639- \\
1.023)\end{array}$ & $1.029(0.782-1.356)$ \\
\hline $\begin{array}{l}30 \text { Sep-12 OctXAdults } \\
\text { w/child(ren) }\end{array}$ & $\begin{array}{c}1.067(0.963- \\
1.182)\end{array}$ & $\begin{array}{c}1.104(0.992- \\
1.230)\end{array}$ & $\begin{array}{l}0.947(0.850- \\
1.055)\end{array}$ & $\begin{array}{c}0.893(0.773- \\
1.031)\end{array}$ & $0.935(0.811-1.077)$ \\
\hline 14-26 OctXSingle adult & $\begin{array}{c}1.012(0.895- \\
1.143)\end{array}$ & $\begin{array}{c}0.936(0.824- \\
1.063)\end{array}$ & $\begin{array}{c}0.977(0.862- \\
1.108)\end{array}$ & $\begin{array}{c}0.962(0.809- \\
1.144)\end{array}$ & $0.906(0.753-1.088)$ \\
\hline 14-26 OctXAdults only & Ref & Ref & Ref & Ref & Ref \\
\hline 14-26 OctXSingle w/ child(ren) & $\begin{array}{c}1.276(1.067- \\
1.526)\end{array}$ & $\begin{array}{c}1.186(0.989- \\
1.423)\end{array}$ & $\begin{array}{c}1.016(0.834- \\
1.236)\end{array}$ & $\begin{array}{c}0.859(0.675- \\
1.093)\end{array}$ & $1.097(0.824-1.460)$ \\
\hline 14-26 OctXAdults w/child(ren) & $\begin{array}{c}1.032(0.930- \\
1.146)\end{array}$ & $\begin{array}{c}1.056(0.946- \\
1.179)\end{array}$ & $\begin{array}{c}1.011(0.901- \\
1.134)\end{array}$ & $\begin{array}{c}1.011(0.861- \\
1.187)\end{array}$ & $0.906(0.774-1.062)$ \\
\hline 28 Oct-9 NovXSingle adult & $\begin{array}{l}1.100(0.964- \\
1.254)\end{array}$ & $\begin{array}{c}1.076(0.938- \\
1.234)\end{array}$ & $\begin{array}{l}0.994(0.863- \\
1.144)\end{array}$ & $\begin{array}{c}1.046(0.865- \\
1.264)\end{array}$ & $1.026(0.837-1.256)$ \\
\hline 28 Oct-9 NovXAdults only & Ref & Ref & Ref & Ref & Ref \\
\hline $\begin{array}{l}28 \text { Oct-9 NovXSingle w/ } \\
\text { child(ren) }\end{array}$ & $\begin{array}{c}1.195(0.975- \\
1.465)\end{array}$ & $\begin{array}{c}1.117(0.908- \\
1.374)\end{array}$ & $\begin{array}{c}0.786(0.642- \\
0.962)\end{array}$ & $\begin{array}{c}0.959(0.745- \\
1.234)\end{array}$ & $0.981(0.740-1.302)$ \\
\hline $\begin{array}{l}28 \text { Oct-9 NovXAdults } \\
\text { w/child(ren) }\end{array}$ & $\begin{array}{c}1.078(0.958- \\
1.213)\end{array}$ & $\begin{array}{c}1.183(1.046- \\
1.338)\end{array}$ & $\begin{array}{c}0.989(0.869- \\
1.127)\end{array}$ & $\begin{array}{c}0.929(0.785- \\
1.099)\end{array}$ & $0.957(0.808-1.135)$ \\
\hline 11-23 NovXSingle adult & $\begin{array}{c}0.994(0.873- \\
1.132)\end{array}$ & $\begin{array}{c}0.965(0.843- \\
1.104)\end{array}$ & $\begin{array}{l}0.920(0.798- \\
1.061)\end{array}$ & $\begin{array}{c}0.905(0.758- \\
1.081)\end{array}$ & $0.916(0.762-1.102)$ \\
\hline 11-23 NovXAdults only & Ref & Ref & Ref & Ref & Ref \\
\hline $\begin{array}{l}\text { 11-23 NovXSingle w/ } \\
\text { child(ren) }\end{array}$ & $\begin{array}{l}1.251(1.041- \\
1.504)\end{array}$ & $\begin{array}{c}1.242(1.029- \\
1.498)\end{array}$ & $\begin{array}{l}0.848(0.680- \\
1.059)\end{array}$ & $\begin{array}{c}0.790(0.603- \\
1.035)\end{array}$ & $1.072(0.788-1.459)$ \\
\hline 11-23 NovXAdults w/child(ren) & $\begin{array}{c}1.033(0.927- \\
1.152)\end{array}$ & $\begin{array}{c}1.095(0.977- \\
1.226)\end{array}$ & $\begin{array}{l}0.966(0.858- \\
1.086)\end{array}$ & $\begin{array}{c}1.015(0.864- \\
1.192)\end{array}$ & $0.897(0.767-1.050)$ \\
\hline Wald p-value & 0.025 & 0.017 & 0.172 & 0.228 & 0.708 \\
\hline Observations & $1,483,378$ & $1,483,378$ & 528,615 & 528,615 & 528,615 \\
\hline
\end{tabular}

Note. OR Odds Ratio. Cl Confidence Intervals. Models also adjust for race/ethnicity, age, sex, household structure, education, cohort, and state of residence. ORs and $\mathrm{Cls}$ in italics significant at $p<.01$. 
Table e8. Logistic regression models with interactions between education and cohort predicting mental health outcomes.

\begin{tabular}{|c|c|c|c|}
\hline Anxiety & Depression & $\begin{array}{l}\text { Prescription } \\
\text { medication }\end{array}$ & $\begin{array}{l}\text { Mental health } \\
\text { services }\end{array}$ \\
\hline
\end{tabular}

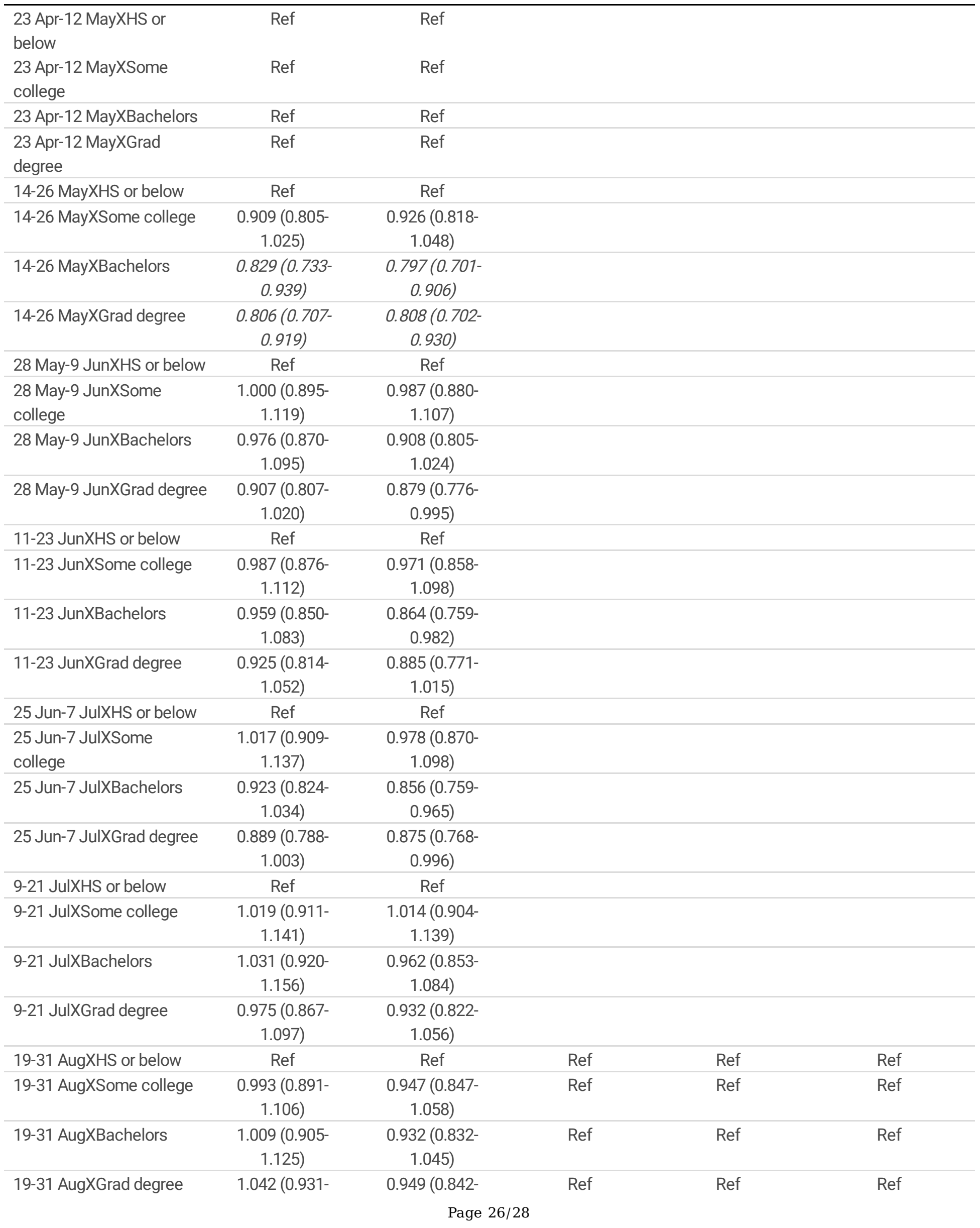


1.166) 1.068)

\begin{tabular}{|c|c|c|c|c|c|}
\hline 2-14 SepXHS or below & Ref & Ref & Ref & Ref & Ref \\
\hline 2-14 SepXSome college & $\begin{array}{c}0.945(0.847- \\
1.055)\end{array}$ & $\begin{array}{c}0.966(0.864- \\
1.081)\end{array}$ & $\begin{array}{c}1.008(0.890- \\
1.142)\end{array}$ & $\begin{array}{c}1.084(0.892- \\
1.318)\end{array}$ & $0.898(0.747-1.079)$ \\
\hline 2-14 SepXBachelors & $\begin{array}{c}0.943(0.845- \\
1.053)\end{array}$ & $\begin{array}{c}0.915(0.815- \\
1.026)\end{array}$ & $\begin{array}{c}0.949(0.836- \\
1.078)\end{array}$ & $\begin{array}{c}0.983(0.811- \\
1.192)\end{array}$ & $0.817(0.678-0.983)$ \\
\hline 2-14 SepXGrad degree & $\begin{array}{c}0.948(0.846- \\
1.061)\end{array}$ & $\begin{array}{c}0.920(0.817- \\
1.036)\end{array}$ & $\begin{array}{c}0.939(0.827- \\
1.066)\end{array}$ & $\begin{array}{c}1.057(0.874- \\
1.278)\end{array}$ & $0.840(0.696-1.013)$ \\
\hline 16-28 SepXHS or below & Ref & Ref & Ref & Ref & Ref \\
\hline 16-28 SepXSome college & $\begin{array}{c}0.974(0.871- \\
1.090)\end{array}$ & $\begin{array}{c}0.968(0.863- \\
1.085)\end{array}$ & $\begin{array}{c}1.095(0.966- \\
1.241)\end{array}$ & $\begin{array}{c}1.225(1.007- \\
1.492)\end{array}$ & $0.967(0.796-1.176)$ \\
\hline 16-28 SepXBachelors & $\begin{array}{c}1.028(0.918- \\
1.151)\end{array}$ & $\begin{array}{c}1.008(0.896- \\
1.135)\end{array}$ & $\begin{array}{c}1.015(0.893- \\
1.153)\end{array}$ & $\begin{array}{c}1.047(0.861- \\
1.273)\end{array}$ & $0.941(0.772-1.146)$ \\
\hline 16-28 SepXGrad degree & $\begin{array}{c}1.031(0.917- \\
1.158)\end{array}$ & $\begin{array}{c}0.968(0.856- \\
1.095)\end{array}$ & $\begin{array}{c}0.994(0.874- \\
1.131)\end{array}$ & $\begin{array}{c}1.127(0.928- \\
1.370)\end{array}$ & $0.876(0.717-1.071)$ \\
\hline $\begin{array}{l}30 \text { Sep-12 OctXHS or } \\
\text { below }\end{array}$ & Ref & Ref & Ref & Ref & Ref \\
\hline $\begin{array}{l}30 \text { Sep-12 OctXSome } \\
\text { college }\end{array}$ & $\begin{array}{c}1.005(0.897- \\
1.126)\end{array}$ & $\begin{array}{c}1.005(0.895- \\
1.129)\end{array}$ & $\begin{array}{c}1.053(0.926- \\
1.197)\end{array}$ & $\begin{array}{c}1.358(1.111- \\
1.658)\end{array}$ & $1.071(0.886-1.294)$ \\
\hline 30 Sep-12 OctXBachelors & $\begin{array}{c}0.993(0.885- \\
1.113)\end{array}$ & $\begin{array}{c}0.923(0.820- \\
1.039)\end{array}$ & $\begin{array}{c}1.007(0.885- \\
1.145)\end{array}$ & $\begin{array}{c}1.191(0.980- \\
1.449)\end{array}$ & $1.081(0.893-1.307)$ \\
\hline $\begin{array}{l}30 \text { Sep-12 OctXGrad } \\
\text { degree }\end{array}$ & $\begin{array}{c}1.023(0.909- \\
1.150)\end{array}$ & $\begin{array}{c}0.918(0.811- \\
1.038)\end{array}$ & $\begin{array}{c}1.033(0.907- \\
1.176)\end{array}$ & $\begin{array}{c}1.292(1.063- \\
1.570)\end{array}$ & $0.955(0.787-1.160)$ \\
\hline 14-26 OctXHS or below & Ref & Ref & Ref & Ref & Ref \\
\hline 14-26 OctXSome college & $\begin{array}{c}0.979(0.872- \\
1.100)\end{array}$ & $\begin{array}{c}0.936(0.832- \\
1.054)\end{array}$ & $\begin{array}{c}1.128(0.982- \\
1.294)\end{array}$ & $\begin{array}{c}1.225(0.980- \\
1.531)\end{array}$ & $0.927(0.751-1.143)$ \\
\hline 14-26 OctXBachelors & $\begin{array}{c}0.991(0.882- \\
1.114)\end{array}$ & $\begin{array}{c}0.907(0.804- \\
1.024)\end{array}$ & $\begin{array}{c}1.073(0.934- \\
1.232)\end{array}$ & $\begin{array}{c}1.021(0.820- \\
1.272)\end{array}$ & $0.882(0.714-1.090)$ \\
\hline 14-26 OctXGrad degree & $\begin{array}{c}0.949(0.842- \\
1.070)\end{array}$ & $\begin{array}{c}0.866(0.764- \\
0.982)\end{array}$ & $\begin{array}{c}1.036(0.900- \\
1.192)\end{array}$ & $\begin{array}{c}1.119(0.898- \\
1.394)\end{array}$ & $0.852(0.687-1.057)$ \\
\hline 28 Oct-9 NovXHS or below & Ref & Ref & Ref & Ref & Ref \\
\hline $\begin{array}{l}28 \text { Oct- } 9 \text { NovXSome } \\
\text { college }\end{array}$ & $\begin{array}{c}0.977(0.857- \\
1.115)\end{array}$ & $\begin{array}{c}0.910(0.796- \\
1.039)\end{array}$ & $\begin{array}{c}1.055(0.904- \\
1.232)\end{array}$ & $\begin{array}{c}1.298(1.027- \\
1.641)\end{array}$ & $0.848(0.684-1.052)$ \\
\hline 28 Oct-9 NovXBachelors & $\begin{array}{c}0.959(0.841- \\
1.095)\end{array}$ & $\begin{array}{c}0.785(0.685- \\
0.899)\end{array}$ & $\begin{array}{c}0.986(0.843- \\
1.153)\end{array}$ & $\begin{array}{c}1.113(0.886- \\
1.398)\end{array}$ & $0.770(0.620-0.956)$ \\
\hline 28 Oct-9 NovXGrad degree & $\begin{array}{c}1.051(0.917- \\
1.205)\end{array}$ & $\begin{array}{c}0.841(0.729- \\
0.971)\end{array}$ & $\begin{array}{c}1.010(0.862- \\
1.184)\end{array}$ & $\begin{array}{c}1.098(0.873- \\
1.381)\end{array}$ & $0.806(0.644-1.010)$ \\
\hline 11-23 NovXHS or below & Ref & Ref & Ref & Ref & Ref \\
\hline 11-23 NovXSome college & $\begin{array}{c}0.979(0.866- \\
1.105)\end{array}$ & $\begin{array}{c}0.976(0.863- \\
1.104)\end{array}$ & $\begin{array}{c}0.954(0.828- \\
1.098)\end{array}$ & $\begin{array}{c}1.255(0.998- \\
1.577)\end{array}$ & $0.953(0.777-1.170)$ \\
\hline 11-23 NovXBachelors & $\begin{array}{c}0.942(0.833- \\
1.066)\end{array}$ & $\begin{array}{c}0.912(0.804- \\
1.035)\end{array}$ & $\begin{array}{c}0.948(0.821- \\
1.094)\end{array}$ & $\begin{array}{c}1.149(0.916- \\
1.442)\end{array}$ & $0.896(0.730-1.101)$ \\
\hline 11-23 NovXGrad degree & $\begin{array}{c}0.924(0.814- \\
1.048) \\
\end{array}$ & $\begin{array}{c}0.870(0.763- \\
0.992) \\
\end{array}$ & $\begin{array}{c}0.931(0.804- \\
1.077) \\
\end{array}$ & $\begin{array}{c}1.217(0.969- \\
1.529) \\
\end{array}$ & $0.841(0.679-1.042)$ \\
\hline Wald p-value & 0.000 & 0.002 & 0.794 & 0.288 & 0.361 \\
\hline Observations & $1,483,378$ & $1,483,378$ & 528,615 & 528,586 & 528,925 \\
\hline
\end{tabular}

Note. OR Odds Ratio. Cl Confidence Intervals. Models also adjust for race/ethnicity, age, sex, household structure, education, cohort, and state of residence. ORs and $\mathrm{Cls}$ in italics significant at $p<.01$ 
Figures

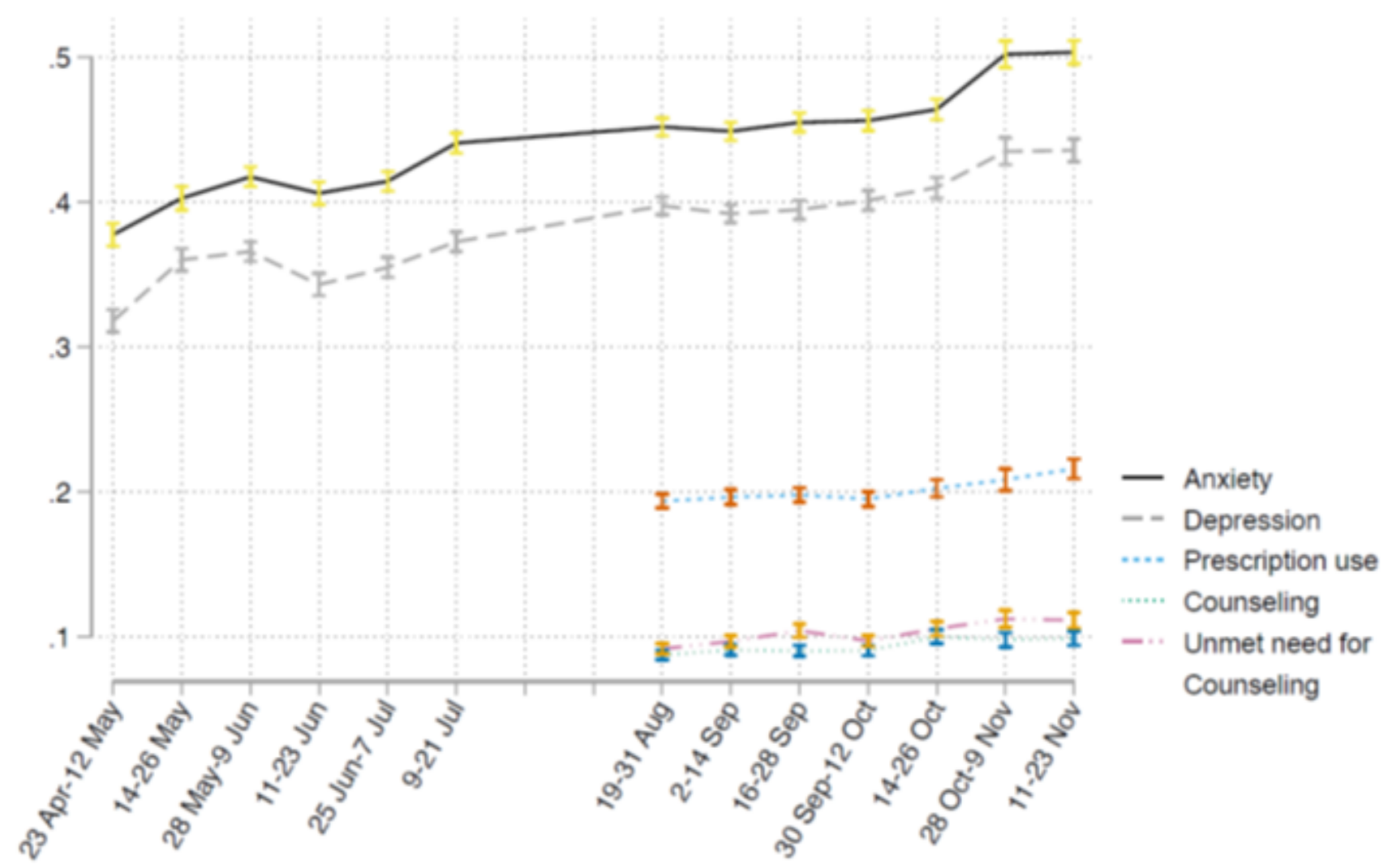

Figure 1

Adjusted prevalence of anxiety, depression, prescription use, mental health counseling, and unmet need for counseling, April-November, 2020. 